\title{
Inertia-Gravity Waves Breaking in the Middle Atmosphere at High Latitudes: Energy Transfer and Dissipation Tensor Anisotropy 0
}

\author{
Tiago Pestana, Matthias Thalhammer, and Stefan Hickel \\ Aerodynamics Group, Faculty of Aerospace Engineering, Technische Universiteit Delft, Delft, Netherlands
}

(Manuscript received 23 December 2019, in final form 18 June 2020)

\begin{abstract}
We present direct numerical simulations of inertia-gravity waves breaking in the middle-upper mesosphere. We consider two different altitudes, which correspond to the Reynolds number of 28647 and 114591 based on wavelength and buoyancy period. While the former was studied by Remmler et al., it is here repeated at a higher resolution and serves as a baseline for comparison with the high-Reynoldsnumber case. The simulations are designed based on the study of Fruman et al., and are initialized by superimposing primary and secondary perturbations to the convectively unstable base wave. Transient growth leads to an almost instantaneous wave breaking and secondary bursts of turbulence. We show that this process is characterized by the formation of fine flow structures that are predominantly located in the vicinity of the wave's least stable point. During the wave breakdown, the energy dissipation rate tends to be an isotropic tensor, whereas it is strongly anisotropic in between the breaking events. We find that the vertical kinetic energy spectra exhibit a clear 5/3 scaling law at instants of intense energy dissipation rate and a cubic power law at calmer periods. The term-by-term energy budget reveals that the pressure term is the most important contributor to the global energy budget, as it couples the vertical and the horizontal kinetic energy. During the breaking events, the local energy transfer is predominantly from the mean to the fluctuating field and the kinetic energy production is in balance with the pseudo kinetic energy dissipation rate.
\end{abstract}

\section{Introduction}

The inherent multiscale nature of atmospheric phenomena makes accurate numerical weather predictions a challenging task. At least for the near feature, solving the complete set of governing equations is out of reach (Bauer et al. 2015). Parameterization techniques, either based on a simplified physical description of the atmospheric processes or based on statistics (e.g., stochastic parameterization; Berner et al. 2017) will therefore continue to gain attention. Nevertheless, steady progress in this area requires unveiling the rationale small-scale atmospheric phenomena. In this regard, direct numerical simulations (DNS) of simplified scenarios can help us understand at least part of the problem.

Supplemental information related to this paper is available at the Journals Online website: https://doi.org/10.1175/JAS-D-190342.s1.

Corresponding author: Tiago Pestana,t.pestana@tudelft.nl
Among the myriad of processes that take place in the atmosphere, gravity waves have been recognized as an important player for the middle and upper atmosphere. One interesting aspect is that due to instabilities they can break and induce turbulence and dissipation in the overall large-scale flow (gravity wave drag). Further, gravity waves transport energy and momentum from where they are created to regions far away. Typical wavelengths of gravity waves are usually unresolved by the numerical grid, which implies that their effects have to be included in simulations through parameterization. For example, the deposition of momentum and heat dissipation in the large-scale flow are usually based on linear wave theory models, such as those originally introduced by Lindzen (1981), or based on nonlinear wave interactions, as in the Doppler-spread parameterization of Hines (1997).

Several studies have focused on gravity waves giving special attention to the growth of instabilities and the breakdown process. The studies of Andreassen et al. (1994), Fritts et al. (1994), and Isler et al. (1994), for instance, were among the first to recognize the full 
three-dimensional character of the breakdown process and to investigate the structure of the eddy motion, as well as its influence on the transport of momentum and heat. More recently, the focus has been shifted toward expanding the parameter space, while fully resolving the smallest turbulent scales. The DNS by Fritts et al. (2009a,b) considered two gravity waves above and below the threshold for static instability and at Reynolds number $\mathscr{O}\left(10^{4}\right)$ (based on the wavelength and the buoyancy period), and Fritts et al. (2013) and Fritts and Wang (2013) analyzed the breakdown of a monochromatic wave due to interaction with vertically varying fine structure.

Whereas most studies like the ones cited above have focused on high-frequency inertia-gravity waves (HGWs) for which the effects of the Coriolis force can be neglected, little attention has been paid to low-frequency inertia-gravity waves (IGWs). In fact, it can be argued that HGWs are more relevant, since the background frequency due to stratification supersedes the Coriolis parameter in the atmosphere. Nevertheless, the dynamics of gravity waves depend on their frequency and must be studied separately (Achatz 2005, 2007a,b).

Regarding the breakdown of IGWs, to the best of our knowledge the work of Remmler et al. (2013) was the first to present resolved three-dimensional simulations. In that work, the authors considered a statically unstable monochromatic wave superimposed with its primary and secondary perturbations derived from the linear theory studies of Achatz (2007b) and Fruman and Achatz (2012). The DNS by Remmler et al. (2013, 2015) showed that the breakdown process constitutes of a sequence of turbulent bursts, accompanied by a rapid reduction of the wave amplitude. Additionally, their analyses indicate that the duration of the breaking events in 3D simulations are shorter than in typical investigations, which assume a three-component velocity field with a two-dimensional spatial dependency (also known as $2.5 \mathrm{D}$ simulations), and that the energy dissipation rate is less homogeneous than for HGWs.

In the present contribution, we build on the work of Remmler et al. (2013) and turn attention to the breaking mechanism of IGWs at higher Reynolds numbers. Our aim is to provide high-fidelity simulation data, which, on the one hand highlight some of the underlying features of the energy conversion during the breakdown process, and, on the other hand, serve as reference data for models that do not resolve inertia-gravity waves and wave-generated turbulence and instead account for their effects through parameterization. Using the same setup as in Fruman et al. (2014) and Remmler et al. (2013), we perform DNS at two distinct Reynolds numbers. We start off with flow-field visualizations describing the breaking events. Second, we investigate the temporal evolution of the kinetic energy and the available potential energy and of their associated energy dissipation rate, as well as the vertical energy spectra. Last, we turn our focus to the energy transfer process. We analyze it from the aspect of the relevance of each term in the energy budget equation, as well as from the view of energy transfers between the mean and the fluctuating field.

\section{Methodology}

\section{a. Governing equations}

We consider fluid motion in Earth's atmosphere governed by the Boussinesq equations on the $f$ plane:

$$
\begin{aligned}
\nabla \cdot \mathbf{u} & =0, \\
\frac{\partial \mathbf{u}}{\partial t}+\mathbf{u} \cdot \nabla \mathbf{u} & =-\nabla p+b \mathbf{n}-f_{c} \mathbf{n} \times \mathbf{u}+\nu \nabla^{2} \mathbf{u}, \\
\frac{\partial b}{\partial t}+\mathbf{u} \cdot \nabla b & =-\mathbf{u} \cdot N^{2} \mathbf{n}+\alpha \nabla^{2} b .
\end{aligned}
$$

Here, $\mathbf{u}=[u, v, w]$ is the velocity field, $t$ denotes time, $p$ is the pressure field, and $b$ is the buoyancy field, which is defined as normalized deviations of the potential temperature $T$ from the vertically averaged temperature $T^{*}(z)$. That is, $b=g\left(T-T^{*}\right) / T_{0}$, where $T_{0}$ and $g$ are the reference temperature and the magnitude of the gravity field, respectively. Further, the properties of the fluid are the kinematic viscosity $\nu$ and the thermal diffusivity $\alpha$, and $f_{c}=2 \Omega_{E} \sin \beta$ is the Coriolis parameter with $\Omega_{E}$ the Earth's angular velocity and $\beta$ the latitude on the $f$ plane. The Brunt-Väisälä frequency is $N^{2}=\left(g / T_{0}\right) d T^{*} / d z$ and $\mathbf{n}$ is the negative gravity unit vector. The coordinate system for a fixed observer on Earth is $\left[x^{\prime}, y^{\prime}, z^{\prime}\right]$.

For vanishing viscosity and thermal diffusivity, Eqs. (1)-(3) admits wavelike solutions of the form $\Re\{[\hat{u}, \hat{v}, \hat{w}, \hat{b}] \exp (I \phi)\}$, where $\Re$ denotes the real part, $\hat{u}, \hat{v}, \hat{w}$, and $\hat{b}$ are complex amplitudes of the velocity and buoyancy field, $\phi$ is the phase (Achatz and Schmitz 2006), and $I$ is the imaginary unit. Here, we will focus on two-dimensional waves that propagate at an angle $\Theta$ with the $x^{\prime}$ direction (see Fig. 1a). In these cases, the gravity wave can be written as

$$
\left[u^{\prime}, v^{\prime}, w^{\prime}, b^{\prime}\right]=\Re\left\{a\left[I \frac{\omega}{k}, \frac{f}{k},-I \frac{\omega}{m},-\frac{N^{2}}{m}\right] e^{I\left(k x^{\prime}+k x^{\prime}-\omega t\right)}\right\},
$$

where $k$ and $m$ are the components of the base wavenumber vector $\mathbf{K}$, such that the wavelength is $\Lambda=2 \pi / K$ 
(a)

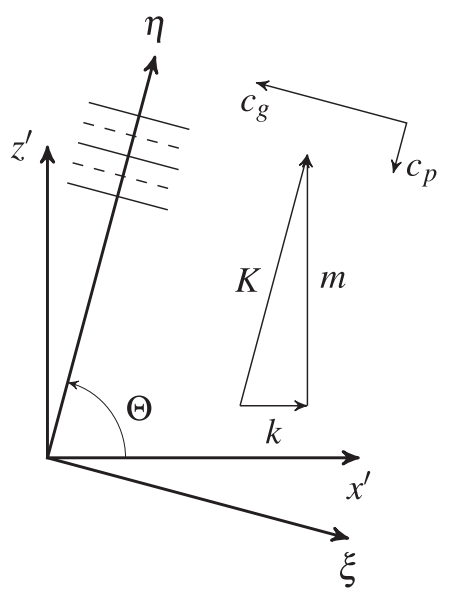

(b)

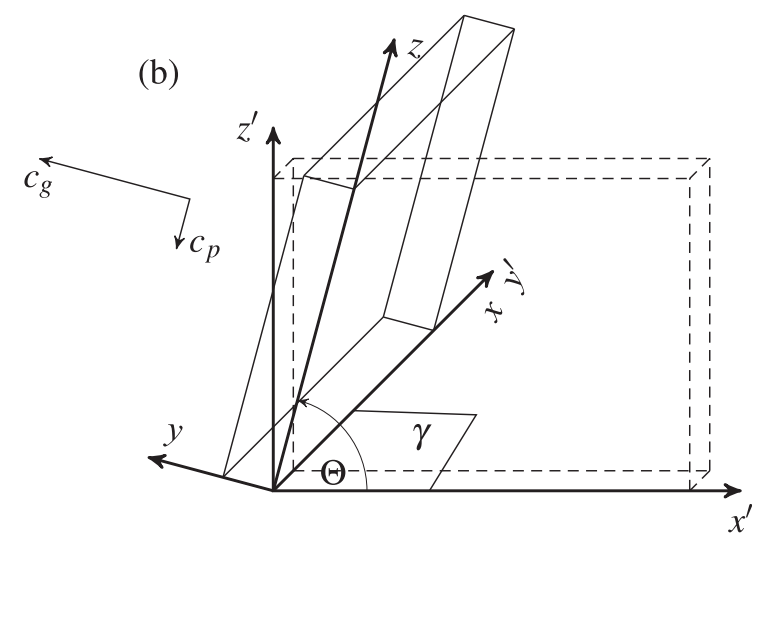

FIG. 1. Sketch showing the relation between the different coordinate systems and the base wave. (a) The rotation of Earth's coordinate system $\left[x^{\prime}, y^{\prime}, z^{\prime}\right]$ about the $y^{\prime}$ axis such that the base wavenumber $\mathbf{K}$ is aligned with the vertical axis $\eta$. (b) The computational domain in the two-times rotated coordinate system $[x, y, z]$.

with $K=\|\mathbf{K}\|=\sqrt{k^{2}+m^{2}}$. In Eq. (4), the wave frequency $\omega$ is defined as

$$
\omega^{2}=N^{2} \cos ^{2} \Theta+f_{c}^{2} \sin ^{2} \Theta,
$$

and $a$ is a nondimensional amplitude. For $a=1$ and $t=0$ the wave is neutrally stable at its least static stable point, i.e., $\partial b / \partial z^{\prime}=-N^{2}$ at $\phi=k x^{\prime}+m z^{\prime}=3 \pi / 2$; see Yau et al. (2004), for example.

We consider inertia-gravity waves with phase velocity $\boldsymbol{c}_{\boldsymbol{p}}$ and group velocity $\boldsymbol{c}_{\boldsymbol{g}}$ as illustrated in Fig. 1 . To ease the representation of the inertia-gravity wave, we introduce a sequence of coordinate transformations, which is better understood by looking at Figs. $1 \mathrm{a}$ and $1 \mathrm{~b}$. The first transformation is a counterclockwise rotation by $\pi / 2-\Theta$ around the positive $y^{\prime}$ direction, such that in the rotated coordinate system $\left(\xi, y^{\prime}, \eta\right)$, the $\eta$ direction is aligned with the wavenumber vector $\mathbf{K}$. The second transformation, on the other hand, simply rearranges the coordinate system through a $\gamma=\pi / 2$ counterclockwise rotation around $\eta$. This set of transformation leads to the double-rotated coordinate system $[x, y, z]$, which is related to Earth's coordinate system $\left[x^{\prime}, y^{\prime}, z^{\prime}\right]$ by

$$
\begin{aligned}
& x^{\prime}=-y \sin \Theta+z \cos \Theta, \\
& y^{\prime}=x, \\
& z^{\prime}=y \cos \Theta+z \sin \Theta .
\end{aligned}
$$

Accordingly, the relation between the velocity field $\left[u^{\prime}, v^{\prime}, w^{\prime}\right]$ in Earth's coordinate frame and the velocity field $[u, v, w]$ in the double-rotated coordinate system is essentially the same as Eq. (6), but with the spatial coordinates replaced by the corresponding velocities, since, e.g., $d x / d t=u$ and $d x^{\prime} / d t=u^{\prime}$. Upon substitution in Eq. (4), and taking the real-valued part only, it can be shown that the monochromatic inertia-gravity wave assumes the following form:

$$
\begin{aligned}
u & =\frac{a f_{c}}{K \cos \Theta} \cos \phi, \\
v & =-\frac{a \omega}{K \sin \Theta \cos \Theta} \sin \phi, \\
w & =0, \\
b & =\frac{a N^{2}}{K \sin \Theta} \cos \phi .
\end{aligned}
$$

Note that $\phi=\left(k x^{\prime}+m z^{\prime}-\omega t\right)=(K z-\omega t)$, which can be obtained with the aid of $k=K \cos \Theta$ and $m=K \sin \Theta$.

\section{b. Numerical setup}

The design of the initial conditions for the simulations uses previous knowledge on the instability of the inertiagravity waves. Following Achatz (2007a), Fruman and Achatz (2012), and Fruman et al. (2014), first a normal mode (NM) analysis of the base wave is performed to identify the perturbation with the largest growth rate. The velocity field of this primary perturbation has three nonzero components that vary along the two spatial directions $x$ and $z$ and which is independent of the third coordinate $y$. Second, a tangent linear stability analysis about the initial 2.5D dynamics developing after perturbing the wave by its leading normal mode is performed to obtain the fully $3 \mathrm{D}$ secondary perturbation, which is taken as the singular vector (SV) that maximizes the perturbation growth within the chosen optimization time (5 min). The initial conditions 


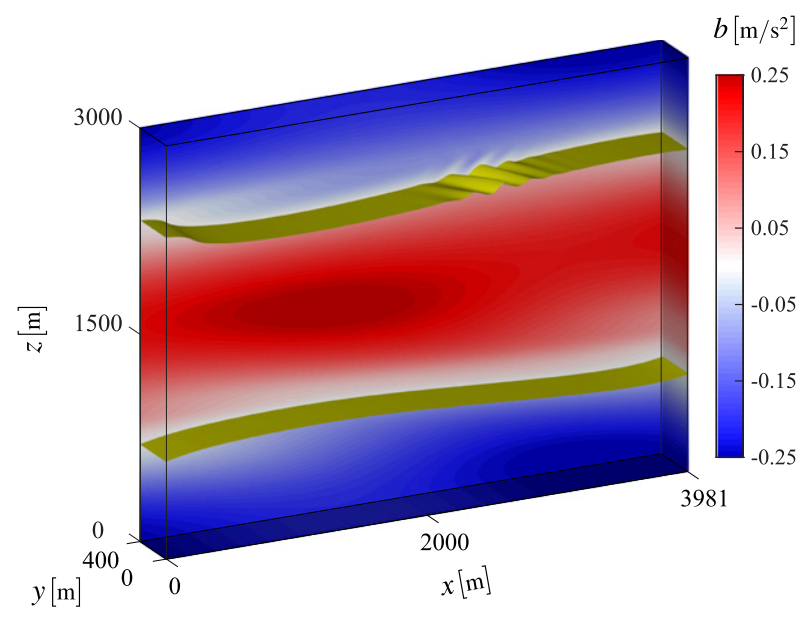

FIG. 2. Contours of the buoyancy field depicting the initial condition for the simulations of cases IGW81 and IGW72. The yellow isosurfaces $(b=0)$ spanning the $x$ and $y$ directions outlines the primary and the secondary perturbations of the base wave.

are then finally composed of the base wave superimposed with its primary (NM) and secondary (SV) perturbations and are fully three-dimensional owing to the singular vector-see Fig. 2 for an illustration of the initial conditions.

Using this technique, we examine two inertia-gravity waves with vertical wavelength $\Lambda=3000 \mathrm{~m}$ propagating at an inclination $\Theta=89.5^{\circ}$ and at different altitudes in the middle atmosphere, namely, at 81 and $72 \mathrm{~km}$. The corresponding horizontal wavelength of the base wave in the Earth frame of reference is $\Lambda_{x^{\prime}}=343 \mathrm{~km}$. The Coriolis parameter is taken from the $f$-plane approximation for a latitude $\beta=70^{\circ}$, i.e., $f_{c}=1.367 \times 10^{-4} \mathrm{~s}^{-1}$, and the Brunt-Väisälä frequency is taken as constant in the range $72-81 \mathrm{~km}$ with $N=2 \times 10^{-2} \mathrm{~s}^{-1}$. In terms of physical parameters, the only difference between the two cases, henceforth referred to as IGW81 and IGW72, is the kinematic viscosity $\nu$, which we estimate from the U.S. standard atmosphere model for the corresponding altitudes. The Reynolds number based on the wavelength of the base wave $\Lambda$ and on the buoyancy period $T_{b}=2 \pi / N$ is $\operatorname{Re}=\Lambda^{2} /\left(\nu T_{b}\right)=28647$ (case IGW81) and $\mathrm{Re}=114591$ (case IGW72). In both cases, the ratio of kinematic viscosity to thermal diffusivity is assumed to be $\operatorname{Pr}=\nu / \alpha=1$, where $\operatorname{Pr}$ is the Prandtl number.

The wavelength of the base wave $(\Lambda)$ defines the extension of the numerical domain in the $z$ direction, whereas the wavelengths of the perturbations, i.e., the leading NM with $\Lambda_{\|}=3981 \mathrm{~m}$ and the SV with $\Lambda_{\perp}=$ $400 \mathrm{~m}$, determines the normal $(x)$ and the transversal $(y)$ domain size: $\left[L_{x}, L_{y}, L_{z}\right]=\left[\Lambda_{\|}, \Lambda_{\perp}, \Lambda\right]=[3981$, 400, 3000] $\mathrm{m}$.

The set of Eqs. (1)-(3) are solved numerically using the pseudospectral technique. To compute the spatial gradients, we employ dealiased ( $2 / 3$ rule) fast Fourier transforms (Pekurovsky 2012). For time integration, we use a third-order low-storage Runge-Kutta scheme to integrate the nonlinear, the Coriolis, and the buoyancy diffusivity terms, whereas the viscous forces are integrated exactly using the integrating factor technique (Rogallo 1977). For comparison, note that in Remmler et al. (2013) the spatial gradients are approximated with a fourth-order finite-volume scheme. Further, as in Remmler et al. (2013), we continuously translate the numerical domain along the direction of wave propagation, i.e., $z$, as if we were following the base wave. The translation velocity equals to the magnitude of the phase velocity.

The numerical tool is essentially the same as in Pestana and Hickel (2019), with little modifications to accommodate the solution of an additional scalar transport equation for the buoyancy field. The total number of grid points is $N_{p}=2304 \times 246 \times 1728$ in case IGW81 and $N_{p}=3072 \times 294 \times 2304$ in case IGW72. The corresponding numerical resolution along the different directions $[\Delta x, \Delta y, \Delta z]$ is $[1.73,1.63,1.74] \mathrm{m}$ for IGW81 and $[1.30,1.36,1.30] \mathrm{m}$ for IGW72.

For a summary of the physical and numerical parameters, please refer to Table 1 .

\section{Results}

Before we present the results, let us first introduce some notations. For any variable, angular brackets represent spatial averages, i.e., $\langle\cdot\rangle_{x y z}$ are box averages, whereas $\langle\cdot\rangle_{x y}$ represent plane averages along the $x$ and $y$ directions. Spatial fluctuations with respect to plane averages are denoted by a prime, e.g., the fluctuation of the velocity in the $x$ direction is $u^{\prime}=$ $u-\langle u\rangle_{x y}$. When convenient, we use indices to denote the different Cartesian directions, i.e., the velocity field $[u, v, w]$ is also referred to as $\left[u_{1}, u_{2}, u_{3}\right]$. Unless stated otherwise, summation over repeated indices is implied.

Cases IGW72 and IGW81 were integrated in time for $6 \mathrm{~h}$. The total simulated time is slightly shorter than the wave period $T_{w}=\Lambda / c_{p}$, which is approximately $8 \mathrm{~h}$. As we will see in the qualitative and quantitative analysis below, the most interesting differences in the breaking behavior of the two cases occur within the simulated first couple of hours.

To assess the sufficiency of the numerical resolution in resolving the smallest scales of turbulence, we estimated the Kolmogorov length scale by computing

$$
\ell_{\mathrm{kolmo}}=\left(\frac{\nu^{3}}{\varepsilon_{k}}\right)^{1 / 4}
$$


TABLE 1. Physical and numerical parameters for the simulation of cases IGW72 and IGW81.

\begin{tabular}{lrl}
\hline \hline Domain size & $L_{x}=3981 \mathrm{~m} ; L_{y}=400 \mathrm{~m} ; L_{z}=3000 \mathrm{~m}$ \\
Wavelength of the base wave & $\Lambda=3000 \mathrm{~m}$ \\
Wavelengths of the perturbations & $\Lambda_{\|}=3981 \mathrm{~m} ; \Lambda_{\perp}=400 \mathrm{~m}$ \\
Wave vector orientation & $=89.5^{\circ}$ \\
$a$ & $=1.2$ \\
Nondimensional wave amplitude & $u=8.97 \mathrm{~m} \mathrm{~s}^{-1} ; v=14.56 \mathrm{~m} \mathrm{~s}^{-1} ; b=0.23 \mathrm{~m} \mathrm{~s}^{-1}$ \\
Base wave amplitude & $c_{p}=-0.106 \mathrm{~m} \mathrm{~s}^{-1}$ \\
Phase velocity & $f_{c}=1.367 \times 10^{-4} \mathrm{~s}^{-1}$ \\
Coriolis parameter & $N=2 \times 10^{-2} \mathrm{~s}^{-1}$ \\
Brunt-Väisälä frequency & \\
Case IGW81 & $\nu=1 \mathrm{~m}^{2} \mathrm{~s}^{-1}$ \\
$\quad$ Kinematic viscosity & $N_{p}=2304 \times 246 \times 1728$ \\
No. of grid points & $\Delta x=1.73 \mathrm{~m} ; \Delta y=1.63 \mathrm{~m} ; \Delta z=1.74 \mathrm{~m}$ \\
$\quad$ Numerical resolution & \\
Case IGW72 & $\nu=0.25 \mathrm{~m}^{2} \mathrm{~s}^{-1}$ \\
$\quad$ Kinematic viscosity & $N_{p}=3072 \times 294 \times 2304$ \\
No. of grid points & $\Delta x=1.30 \mathrm{~m} ; \Delta y=1.36 \mathrm{~m} ; \Delta z=1.30 \mathrm{~m}$ \\
Numerical resolution &
\end{tabular}

locally and for every instant of time. In Eq. (8), $\varepsilon_{k}=$ $2 S_{i j} S_{i j}$ is the local kinetic energy dissipation rate, with $S_{i j}=\left(\partial u_{i} / \partial x_{j}+\partial u_{j} / \partial x_{i}\right) / 2$ the strain-rate tensor. We find that for case IGW81, the numerical resolution guarantees that approximately at all times $\Delta<\pi \ell_{\text {kolmo }}$ (Kaneda et al. 2003), where $\Delta$ is the smallest grid width, i.e., $\Delta=\min \{\Delta x, \Delta y, \Delta z\}$. For case IGW72, the resolution is lower and satisfies, on average, $\Delta<1.43 \pi \ell_{\text {kolmo }}$. We are thus confident that both simulations resolve the small-scale turbulent motion and that the results would remain unchanged if the mesh is further refined.

\section{a. Flow-field description}

A flow-field visualization provides us with the general picture of the problem. In addition to several instantaneous snapshots (Figs. 3 and 4 ), two videos are included as online supplementary material (see Movie 1 and Movie 2 at https://doi.org/10.1175/JAS-D-19-0342.s1). During the course of the simulation, we observe that bursts of turbulence induce small-scale motion in the overall large-scale flow. In both cases, however, turbulence is not omnipresent; it is instead confined to certain regions of the domain and peculiar of specific time intervals. The main qualitative difference between cases IGW81 and IGW72 is the presence of much finer scales of motion in the latter, a natural consequence of its higher Reynolds number.

The primary and secondary perturbations that define the initial conditions cause the wave to break almost instantly in both cases. The breaking events begin with three-dimensional flow structures developing in the upper half of the domain where the base wave is least stable, i.e., $\phi=3 \pi / 2$ for $z=3 \Lambda / 4=$ $2250 \mathrm{~m}$ (cf. section 2a). (Note that the unstable region remains always located in the upper half of the domain, because the coordinate system is continuously translated with the phase speed). Thereupon, the flow structures are continuously transported horizontally and eventually they spread over the entire domain. Although these features are salient during the first hours of the simulation (Figs. 3a-f and 4a-f), this sequence of events also reoccurs at later times, but in weaker intensity.

To identify the actual time and the number of breaking events, we follow the temporal evolution of the total energy dissipation rate:

$$
\left\langle\varepsilon_{t}\right\rangle_{x y z}=2 \nu\left\langle S_{i j} S_{i j}\right\rangle_{x y z}+\frac{\alpha}{N^{2}}\left\langle\frac{\partial b}{\partial x_{i}} \frac{\partial b}{\partial x_{i}}\right\rangle_{x y z},
$$

where the first and the second term on the right-hand side are the kinetic energy dissipation rate $\left\langle\varepsilon_{k}\right\rangle_{x y z}$ and the potential energy dissipation rate $\left\langle\varepsilon_{b}\right\rangle_{x y z}$, respectively.

The total energy dissipation rate increases significantly at the times for which turbulent spots dominate the flow field. By following the time evolution of $\left\langle\varepsilon_{t}\right\rangle_{x y z}$ (Fig. 5), we observe four peaks in $\left\langle\varepsilon_{t}\right\rangle_{x y z}$ for case IGW81, whereas three peaks are seen for case IGW72. By searching for local maxima, we identify the time of each breaking event as $t_{\mathrm{be}}=\{0.44 ; 1.84 ; 4.05$; $4.95\} \mathrm{h}$ for IGW81 and $t_{\mathrm{be}}=\{0.38 ; 1.92 ; 4.88\} \mathrm{h}$ for IGW72. Although the evolution of $\left\langle\varepsilon_{t}\right\rangle_{x y z}$ is alike during the first breakdown for the two Reynolds numbers, differences in magnitude and duration are evident for the remaining breaking events. For example, for the second breaking event, at around $t=$ $1.88 \mathrm{~h},\left\langle\varepsilon_{t}\right\rangle_{x y z}$ is approximately 1.6 times larger for case IGW81 than for case IGW72; the inverse is, however, observed for the last breaking event, at around $t=5 \mathrm{~h}$, 


\begin{tabular}{cccccc}
\multicolumn{7}{c}{$b\left[\mathrm{~m} / \mathrm{s}^{2}\right]$} \\
& -0.15 & -0.05 & 0.05 & 0.15 & 0.25
\end{tabular}

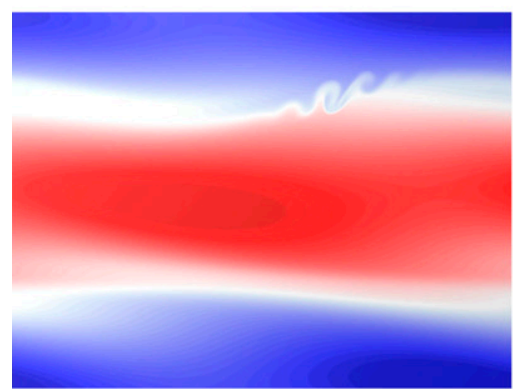

(a) $t=0.03 \mathrm{~h}$

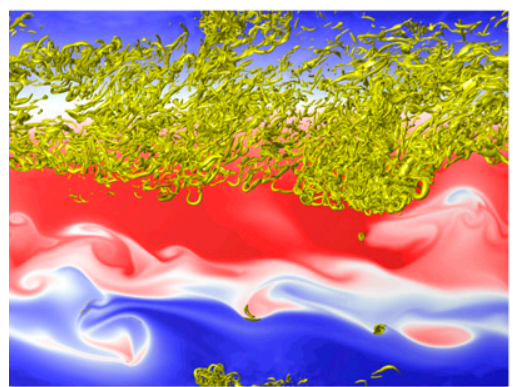

(d) $t=0.34 \mathrm{~h}$

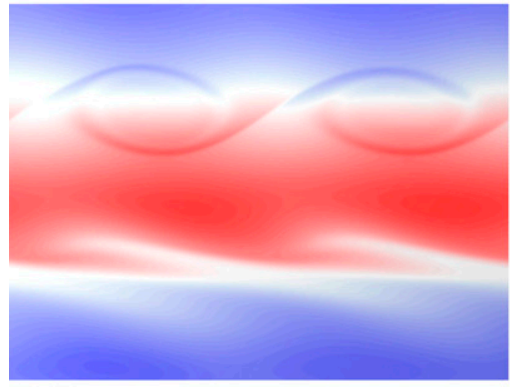

(g) $t=3.68 \mathrm{~h}$

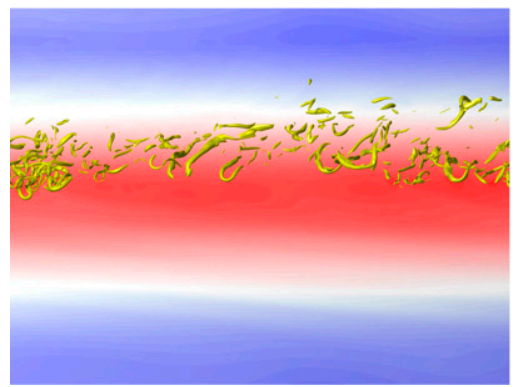

(j) $t=4.64 \mathrm{~h}$

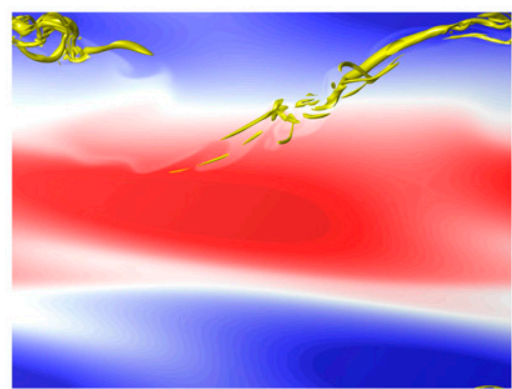

(b) $t=0.09 \mathrm{~h}$

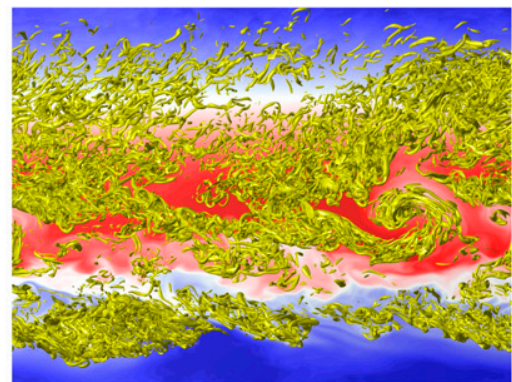

(e) $t=0.47 \mathrm{~h}$

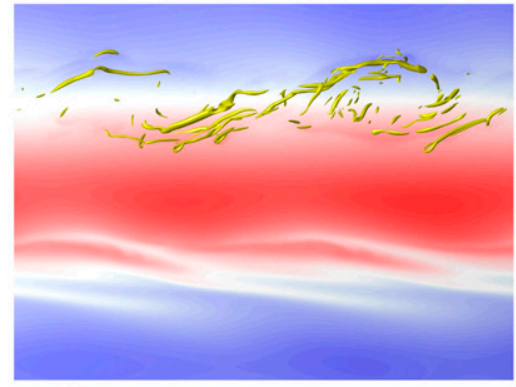

(h) $t=3.94 \mathrm{~h}$

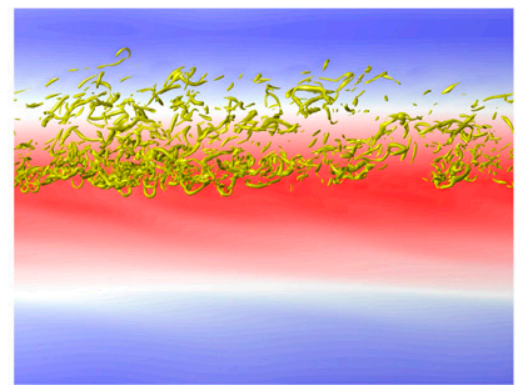

(k) $t=4.96 \mathrm{~h}$

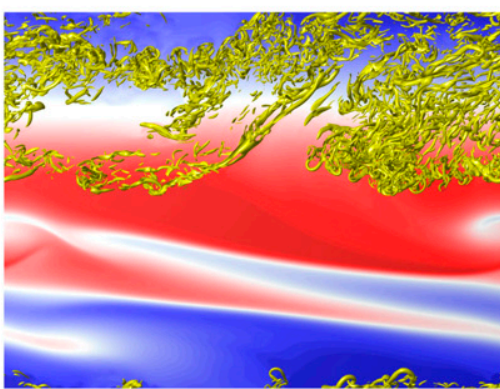

(c) $t=0.24 \mathrm{~h}$

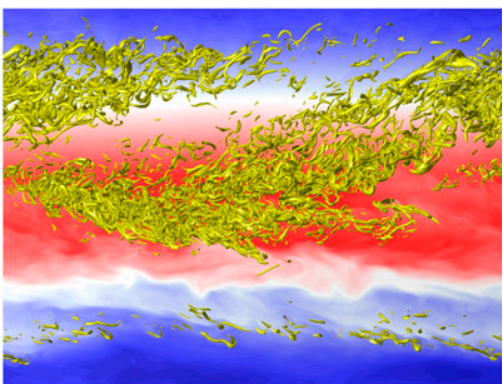

(f) $t=0.57 \mathrm{~h}$

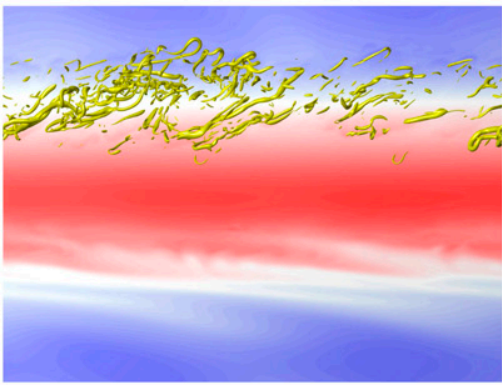

(i) $t=4.12 \mathrm{~h}$

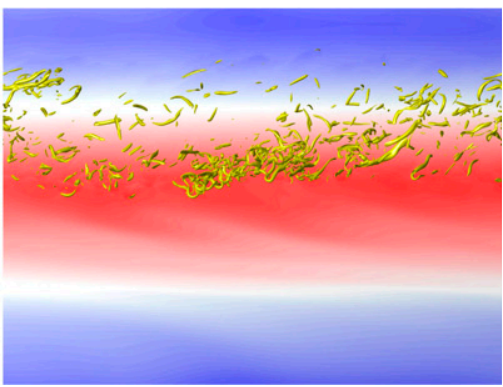

(l) $t=5.12 \mathrm{~h}$

FIG. 3. Instantaneous flow-field visualizations for case IGW81 showing the isosurfaces $Q=0.004 \mathrm{~s}^{2}$ on top of contours of the buoyancy field on the plane $y=400 \mathrm{~m}$. (a)-(f) The first, (g)-(i) third, and (j)-(l) fourth breaking events. 


\begin{tabular}{cccccc}
\multicolumn{9}{c}{$b\left[\mathrm{~m} / \mathrm{s}^{2}\right]$} \\
& -1 & 1 & & \\
\hline & -0.15 & -0.05 & 0.05 & 0.15 & 0.25
\end{tabular}

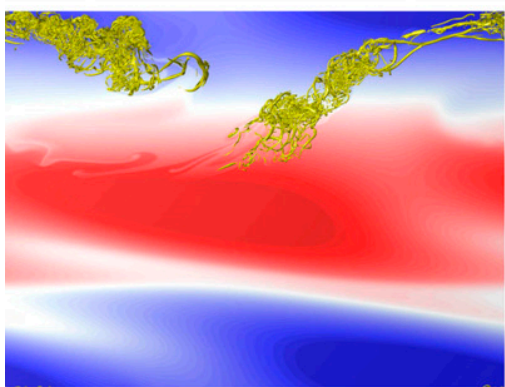

(a) $t=0.10 \mathrm{~h}$

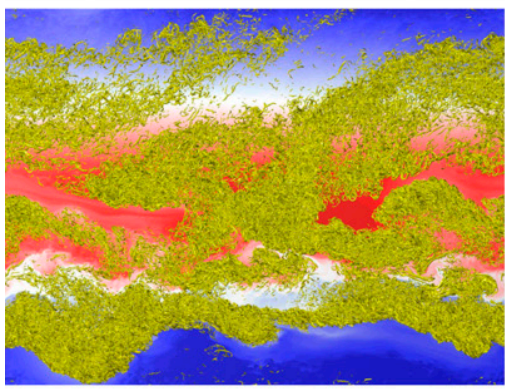

(d) $t=0.42 \mathrm{~h}$

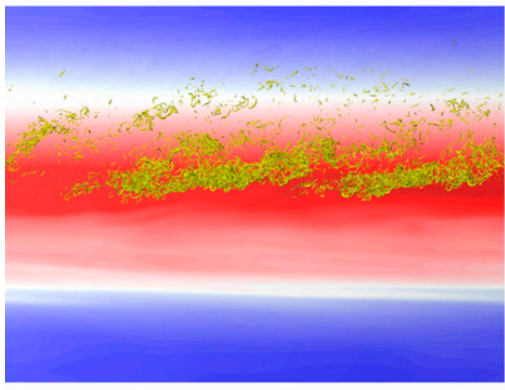

(g) $t=1.85 \mathrm{~h}$

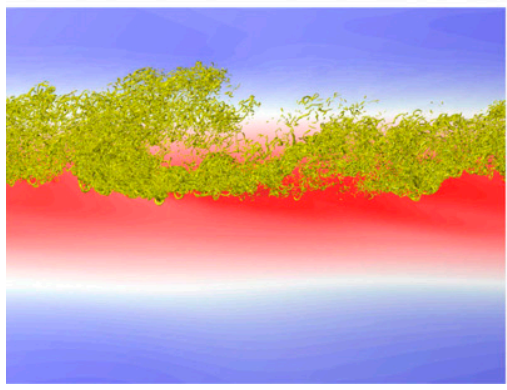

(j) $t=4.79 \mathrm{~h}$

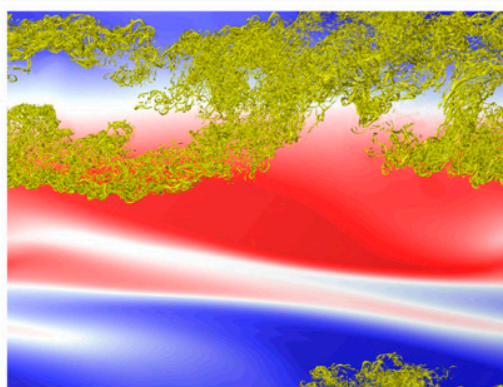

(b) $t=0.19 \mathrm{~h}$

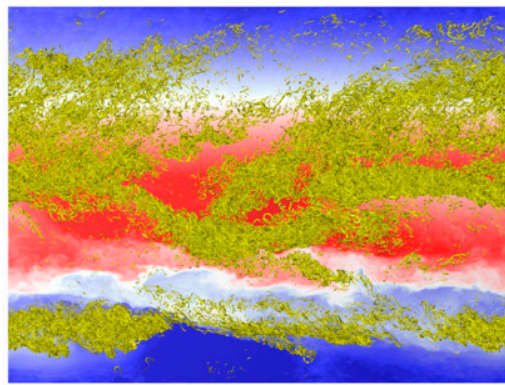

(e) $t=0.48 \mathrm{~h}$

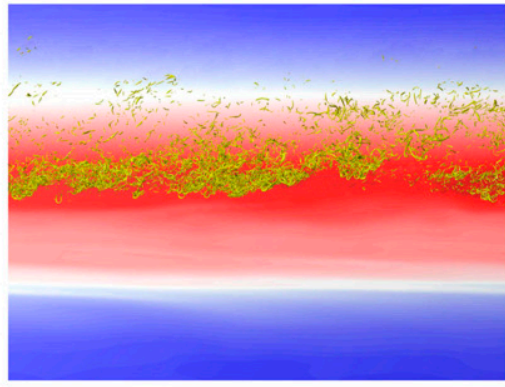

(h) $t=1.94 \mathrm{~h}$

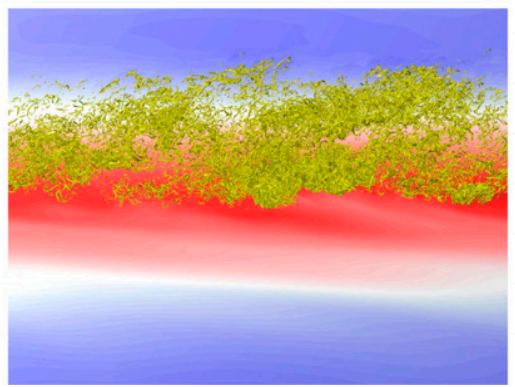

(k) $t=4.92 \mathrm{~h}$

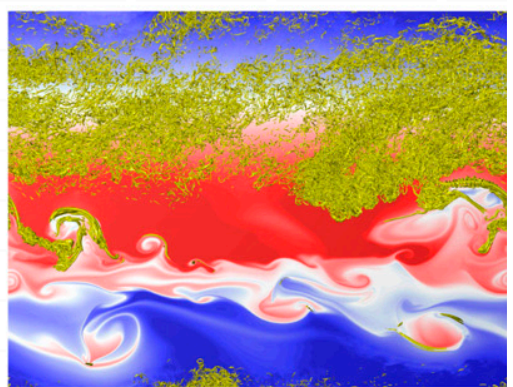

(c) $t=0.33 \mathrm{~h}$

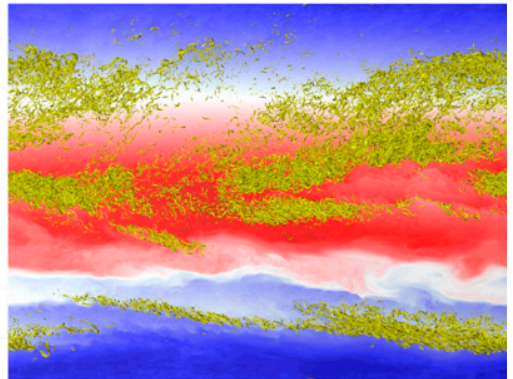

(f) $t=0.59 \mathrm{~h}$

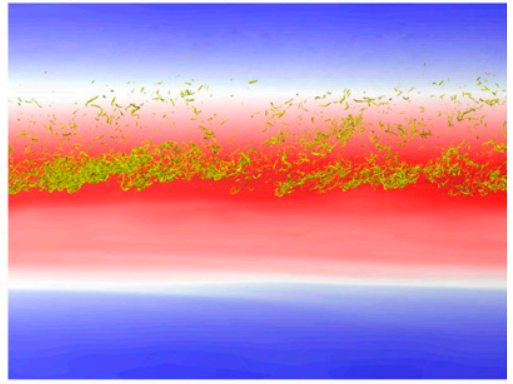

(i) $t=2.04 \mathrm{~h}$

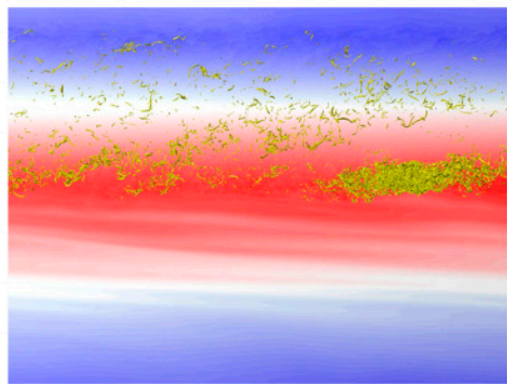

(l) $t=5.36 \mathrm{~h}$

FIG. 4. Instantaneous flow-field visualizations for case IGW72 showing the isosurfaces $Q=0.03 \mathrm{~s}^{2}$ on top of contours of the buoyancy field on the plane $y=400 \mathrm{~m}$. (a)-(f) The first, (g)-(i) second, and (j)-(l) correspond to the and the third breaking events. 


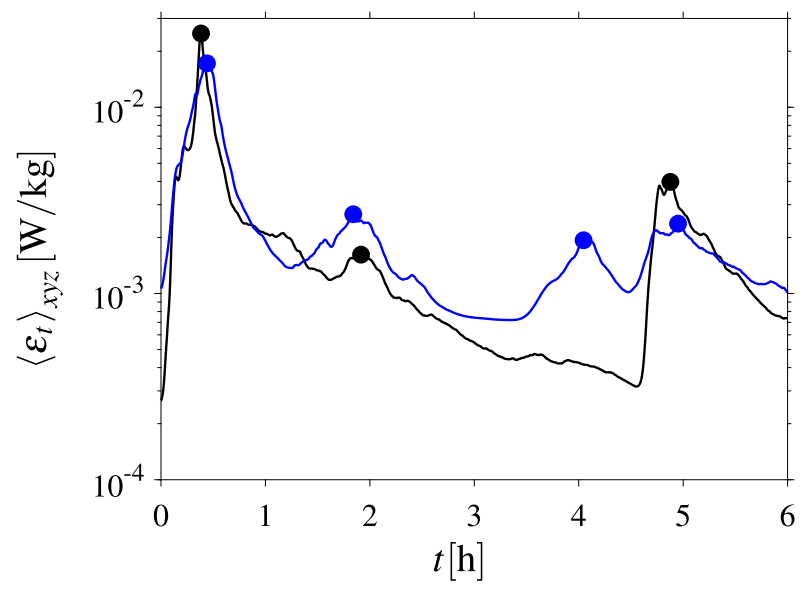

FIG. 5. Time evolution of the total energy dissipation rate $\left\langle\varepsilon_{t}\right\rangle_{x y z}$ for cases IGW81 (blue) and IGW72 (black). The filled circles indicate local maxima and the instant of each breaking event.

where $\left\langle\varepsilon_{t}\right\rangle_{x y z}$ for case IGW72 is larger than for IGW81 by roughly the same amount.

This sequence of breaking events causes the nondimensional wave amplitude $a$ to decay monotonically with time (Fig. 6). For $t \lesssim 0.5 \mathrm{~h}, a(t)$ follows the same trend in both cases and decays rapidly. This is probably a consequence of the well-defined initial conditions, which cause the first breaking event to be similar in both cases. At posterior times, the decay rate reduces significantly and case IGW72 exhibits a slower decay rate, which is expected from a laminar decay: for $\operatorname{Pr}=1$, the nondimensional wave amplitude of an unperturbed laminar wave evolves as $a_{\text {lam }}(t)=a_{0} \exp \left(-\nu K^{2} t\right)$ (Fruman et al. 2014). More interesting, we also notice that the third and fourth breaking for case IGW81 occurs although the nondimensional amplitude is below the limit for static instability, i.e., $a<1$. For case IGW72, $a>1$ for most part of the simulation, but during the last breaking event $a$ is also smaller than 1 .

\section{b. Dissipation tensor anisotropy}

The contributions of the kinetic and the potential energy dissipation rate to $\left\langle\varepsilon_{t}\right\rangle_{x y z}$ [Eq. (9)] differ strongly between both cases and varies over time. In fact, Fig. 7 shows that the main contributor to $\left\langle\varepsilon_{t}\right\rangle_{x y z}$ is $\left\langle\varepsilon_{k}\right\rangle_{x y z}$. By comparing the temporal evolution of the kinetic and potential energy dissipation rates, we find from Figs. $7 \mathrm{a}$ and $7 \mathrm{~b}$ that at the peak of the first breaking event the ratio $\left\langle\varepsilon_{k}\right\rangle_{x y z} /\left\langle\varepsilon_{b}\right\rangle_{x y z}$ is approximately 1.96 irrespective of the Reynolds number. Nevertheless, the disparity between the two quantities increases at later times. For example, at the last breaking event of case IGW72 the ratio is approximately 16.1 .

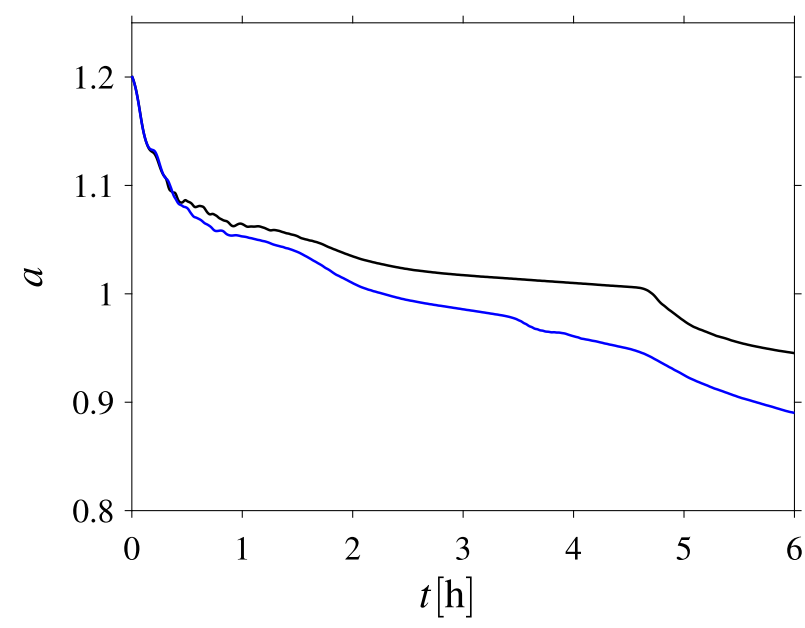

FIG. 6. Time evolution of the nondimensional wave amplitude $a$ for cases IGW81 (blue) and IGW72 (black).

More information regarding the structure of the energy dissipation tensor is obtained by decomposing it in its individual components along the three Cartesian directions, i.e.:

$$
\left\langle\varepsilon_{k}\right\rangle_{x y z}=\frac{1}{2}\left(\left\langle\varepsilon_{k, 11}\right\rangle_{x y z}+\left\langle\varepsilon_{k, 22}\right\rangle_{x y z}+\left\langle\varepsilon_{k, 33}\right\rangle_{x y z}\right) .
$$

By assessing the relative importance of each term on the right-hand side of the equation above (Fig. 8), we find that all three components contribute almost equally to $\left\langle\varepsilon_{k}\right\rangle_{x y z}$ at instants of time corresponding to the wave breakdown. The later observation, however, is more explicit for the higher-Reynolds-number case (Fig. 8b). Although we also observe the same tendency for the lower-Reynolds-number case (Fig. 8a), the difference between the components is still significant. Put together, these results indicate that for increasing Reynolds number, we can expect the energy dissipation tensor to attain an isotropic structure during the phases with high turbulence intensity, while during calmer periods, we can expect the kinetic energy dissipation tensor to have a strongly anisotropic structure. A certainly interesting observation is that the component aligned with the direction of wave propagation has the smallest contribution to the energy dissipation for both cases.

\section{c. Hovmöller diagrams}

Now, we turn our attention to the distribution of different quantities along the direction of wave propagation. In particular, we look at the spatial distribution of $\varepsilon_{k}$ and $\varepsilon_{b}$ and their relation to the Richardson number (Ri). For this purpose, we average the different quantities over planes normal to the direction of propagation to obtain $\left\langle\varepsilon_{k}\right\rangle_{x y},\left\langle\varepsilon_{b}\right\rangle_{x y}$ and $\langle\mathrm{Ri}\rangle_{x y}$. Whereas the kinetic 

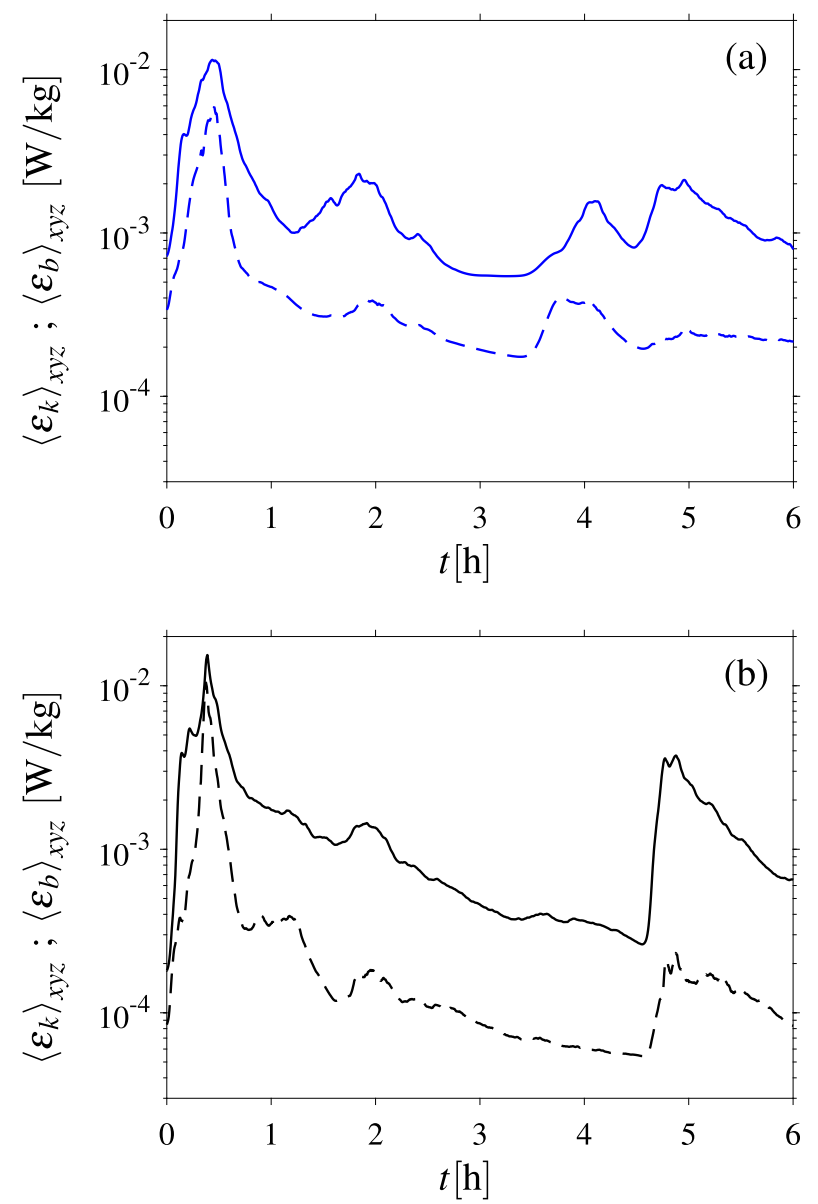

FIG. 7. Time evolution of the kinetic and potential energy dissipation rate for cases (a) IGW81 and (b) IGW72: $\left\langle\varepsilon_{k}\right\rangle_{x y z}$ (solid) and $\left\langle\varepsilon_{b}\right\rangle_{x y z}$ (dashed).

and potential energy dissipation are obtained similarly to Eq. (9), the Richardson number follows from the local balance between buoyancy gradients and shear rate. In Earth's frame of reference, it is given as

$$
\operatorname{Ri}=\frac{N^{2}+\left(\partial b / \partial z^{\prime}\right)}{\left(\partial u / \partial z^{\prime}\right)^{2}+\left(\partial v / \partial z^{\prime}\right)^{2}} .
$$

The Richardson number is computed in the rotated frame of reference $[x, y, z]$ by projection of the gradients.

A first hint toward the spatial distribution of the energy dissipation rate can be obtained from the previous flow-field visualization. For instance, a comparison of the first breaking event in both cases (Figs. 3a-f and $4 \mathrm{a}-\mathrm{f}$ ) shows that the regions of stronger spatial gradients in the buoyancy field (lower half of the domain cf. Fig. 3c) do not coincide with the location of the eddies. In the upper half of the domain, the isopycnals are primarily horizontal and neatly separated from each other, whereas the lower half of the domain shows
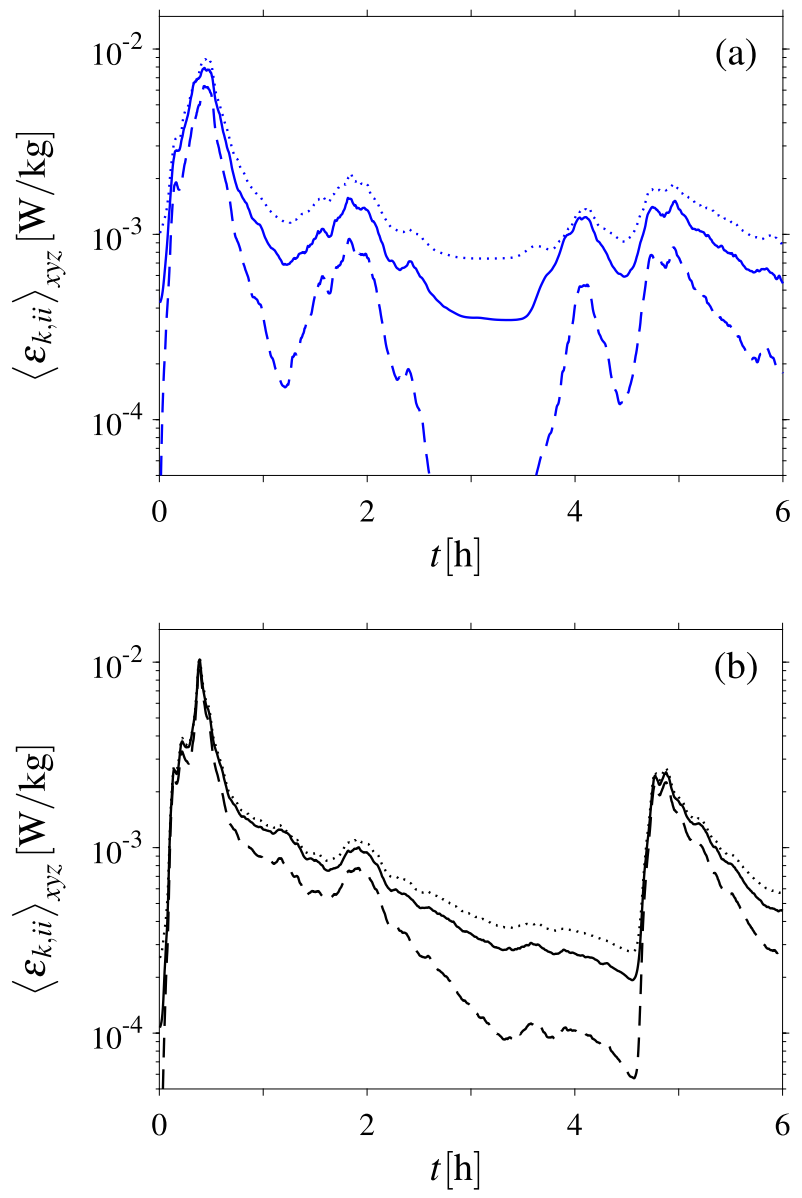

FIG. 8. Time evolution of the components of the kinetic energy dissipation-rate tensor for cases (a) IGW81 and (b) IGW72: $\left\langle\varepsilon_{k, 11}\right\rangle_{x y z}$ (solid), $\left\langle\varepsilon_{k, 22}\right\rangle_{x y z}$ (dotted), and $\left\langle\varepsilon_{k, 33}\right\rangle_{x y z}$ (dashed).

regions of intense mixing in the buoyancy field and consequently larger spatial gradients.

To confirm these observations, we present in Fig. 9 Hovmöller diagrams of $\left\langle\varepsilon_{k}\right\rangle_{x y},\left\langle\varepsilon_{b}\right\rangle_{x y}$ and $\langle\mathrm{Ri}\rangle_{x y}$, i.e., their evolution as a function of $z$ and $t$. From Figs. 9a and 9d, we see that large values of $\left\langle\varepsilon_{k}\right\rangle_{x y}$ dominate the region $z>1.5 \mathrm{~km}$ and persist in this location for the entire simulation time. On the contrary, Figs. $9 \mathrm{~b}$ and $9 \mathrm{e}$ show that high dissipation of potential energy is found for $z<$ $1.5 \mathrm{~km}$ and it is restricted to $t<2 \mathrm{~h}$. Overall, we see that the upper half of the domain is marked by a higher level of turbulence activity (stronger spatial gradients), in agreement with the visualization of finer scales in the same region.

Regardless of the Reynolds number, the regions of intense dissipation of kinetic energy are more likely to be found within an envelope delimited by the stability threshold, i.e., $\langle\mathrm{Ri}\rangle_{x y}=0.25$. Figures $9 \mathrm{c}$ and $9 \mathrm{f}$ show that values for which $\langle\mathrm{Ri}\rangle_{x y}<0.25$ are predominantly located in the upper half of the domain, thus evidencing 

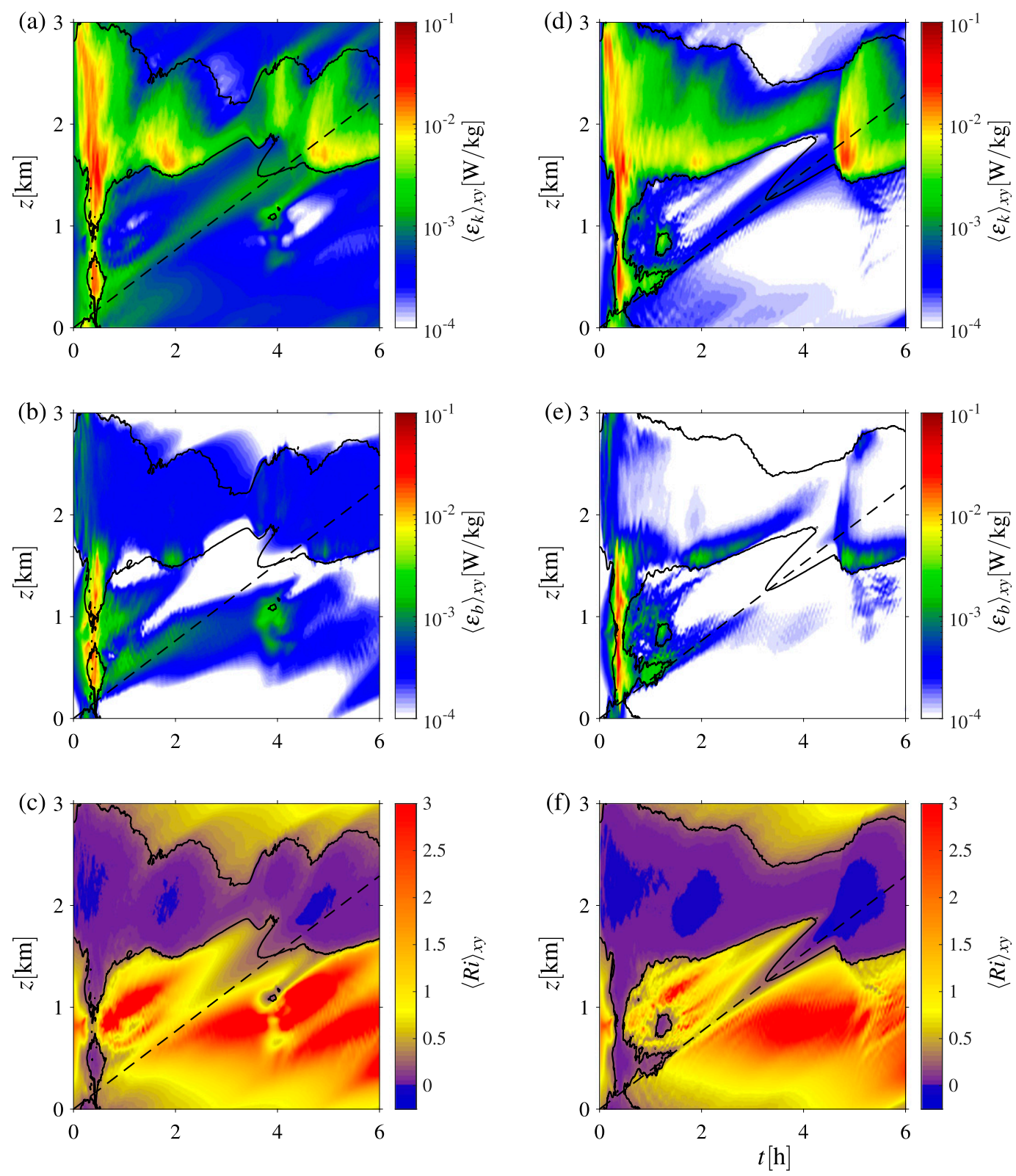

FIG. 9. Hovmöller plots of the (top) kinetic and (middle) potential energy dissipation rates, and (bottom) the Richardson number. Cases (a)-(c) IGW81 and (d)-(f) IGW72. The black solid line represents the contour line Ri = 0.25 , and the dashed line tracks a fixed position in Earth's frame of reference.

the unstable nature of the upper half of the wave domain. For comparison, we superimpose the contour $\langle\mathrm{Ri}\rangle_{x y}=$ 0.25 on the distribution of $\left\langle\varepsilon_{k}\right\rangle_{x y}$ and $\left\langle\varepsilon_{b}\right\rangle_{x y}$-see Figs. 9a, $9 \mathrm{~b}, 9 \mathrm{~d}$, and $9 \mathrm{e}$. We observe that $\langle\mathrm{Ri}\rangle_{x y}=0.25$ delineates well the region of high kinetic energy dissipation, indicating a correlation between both quantities.

For these results, there are no significant differences between cases IGW81 and IGW72, apart from the fact that in case IGW72, the regions of intense kinetic energy dissipation are broader and better defined than in case
IGW81. Incidentally, as observed by Remmler et al. (2013) for case IGW81, Figs. 9a and 9b also show that in both cases the last breaking event occurs when remnant turbulence from the first breaking event meets the least unstable region of the base wave. This observation follows from tracking the evolution of a fixed point in Earth's frame of reference, as represented by the dashed line in that figure. Although case IGW81 shows a clearer region of somewhat high kinetic energy dissipation traveling with the phase speed in the comoving frame of 

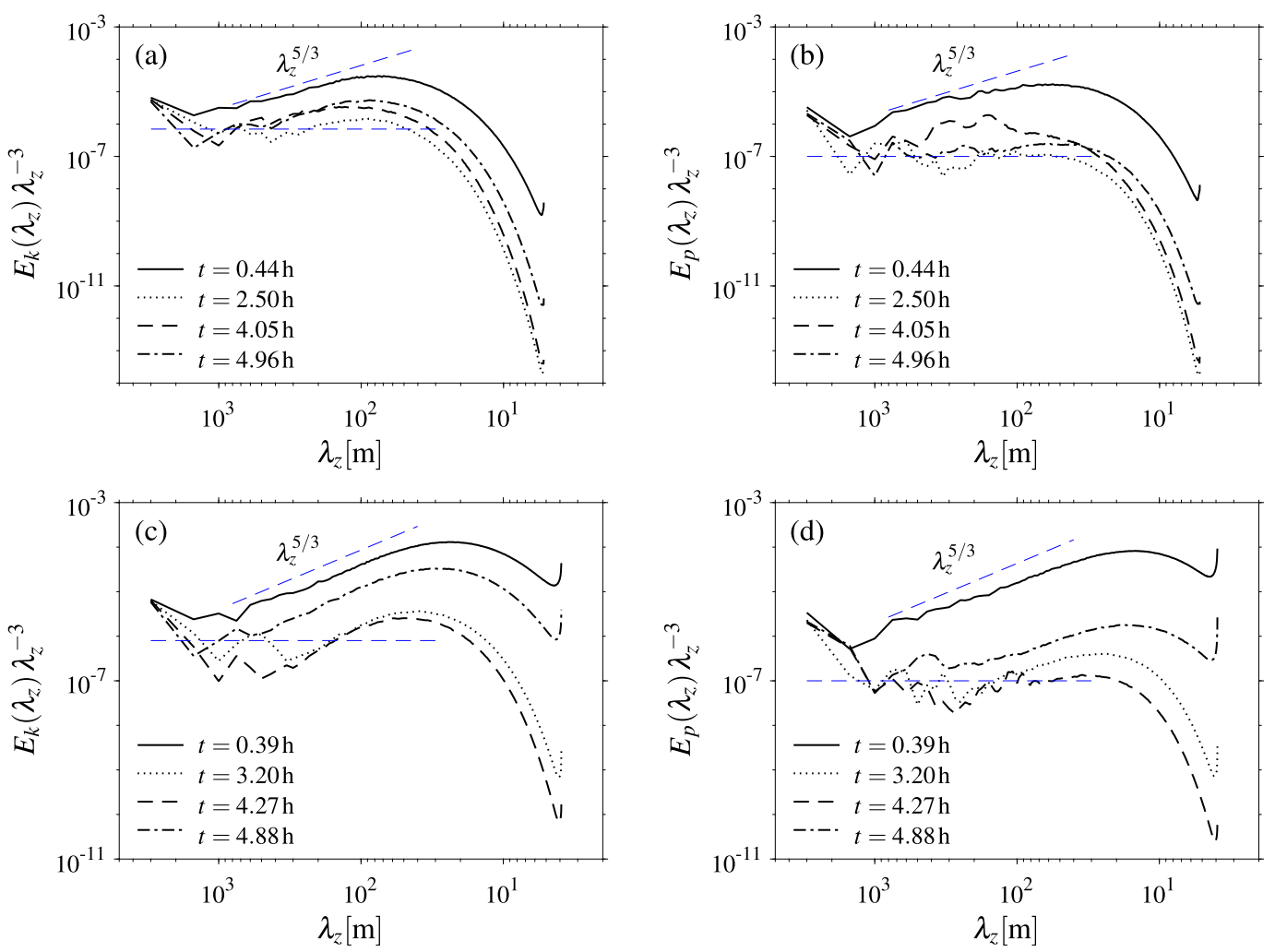

FIG. 10. Compensated (left) kinetic and (right) potential energy spectra plotted over the vertical wavelength $\lambda_{z}$ for different time instants. (a),(b) Case IGW81. (c),(d) Case IGW72. The thin dashed blue lines indicates the $\lambda_{z}^{5 / 3}$ and $\lambda_{z}^{3}$ scaling laws.

reference, case IGW72 indicates that an even lower small-scale turbulence activity might be already sufficient to trigger instabilities and be amplified.

\section{d. Energy spectra}

The distinct turbulence level characteristic of the different time instants is also reflected in both the kinetic and potential energy spectra. In Fig. 10 we show the spectra in terms of the vertical wavelength $\lambda_{z}$ for both cases. The kinetic and potential energy spectra, $E_{k}\left(\lambda_{z}\right)$ and $E_{p}\left(\lambda_{z}\right)$, respectively, are defined such that

$$
\int_{\lambda_{z}} E_{k}\left(\lambda_{z}\right) d \lambda_{z}=\langle K\rangle_{x y z}
$$

and

$$
\int_{\lambda_{z}} E_{p}\left(\lambda_{z}\right) d \lambda_{z}=\langle A\rangle_{x y z}
$$

where $K=\left(u_{i} u_{i}\right) / 2$ and $A=b^{2} /\left(2 N^{2}\right)$ are the local kinetic and potential energy. For the following comparison of the energy spectra, we intentionally select two time instants during the breaking event and two additional ones at calmer periods, which correspond to off-peak locations in the evolution of $\left\langle\varepsilon_{t}\right\rangle_{x y z}$.

At time instants corresponding to the breaking events, $E_{k}\left(\lambda_{z}\right)$ and $E_{p}\left(\lambda_{z}\right)$ scales in good agreement with $\lambda_{z}^{5 / 3}$. For instance, in case IGW72 (Figs. 10c,d) we observe that for $t=0.39 \mathrm{~h}$ and $t=4.88 \mathrm{~h}$, i.e., approximately during the first and the third breaking events, respectively, $E_{k}\left(\lambda_{z}\right)$ and $E_{p}\left(\lambda_{z}\right)$ exhibits a $\lambda_{z}^{5 / 3}$ scaling from $\lambda_{z}=$ $37 \mathrm{~m}$ to $\lambda_{z}=750 \mathrm{~m}$. On the other hand, the same figure shows that quiescent periods, e.g., $t=3.20$ and $4.27 \mathrm{~h}$, the scaling of the energy spectra matches better a $\lambda_{z}^{3}$ law. Put together, these results suggest that during the breaking events the flow field is isotropic, and follows a Kolmogorov scaling of the type $\lambda_{z}^{5 / 3}$, which is canonical for homogeneous isotropic turbulence. Further, the $\lambda_{z}^{3}$ scaling at calmer periods indicates that buoyancy effects are more relevant during periods of decay rather than during the periods of intense wave breaking, since a $\lambda_{z}^{3}$ is expected in the inertial-range of strongly stratified flows (Brethouwer et al. 2007; Remmler and Hickel 2013).

Although the power-law dependencies are best seen in case IGW72 (Figs. 10c,d), their footprint is also clear in case IGW81 (Figs. 10a,b). Therefore, we hypothesize 

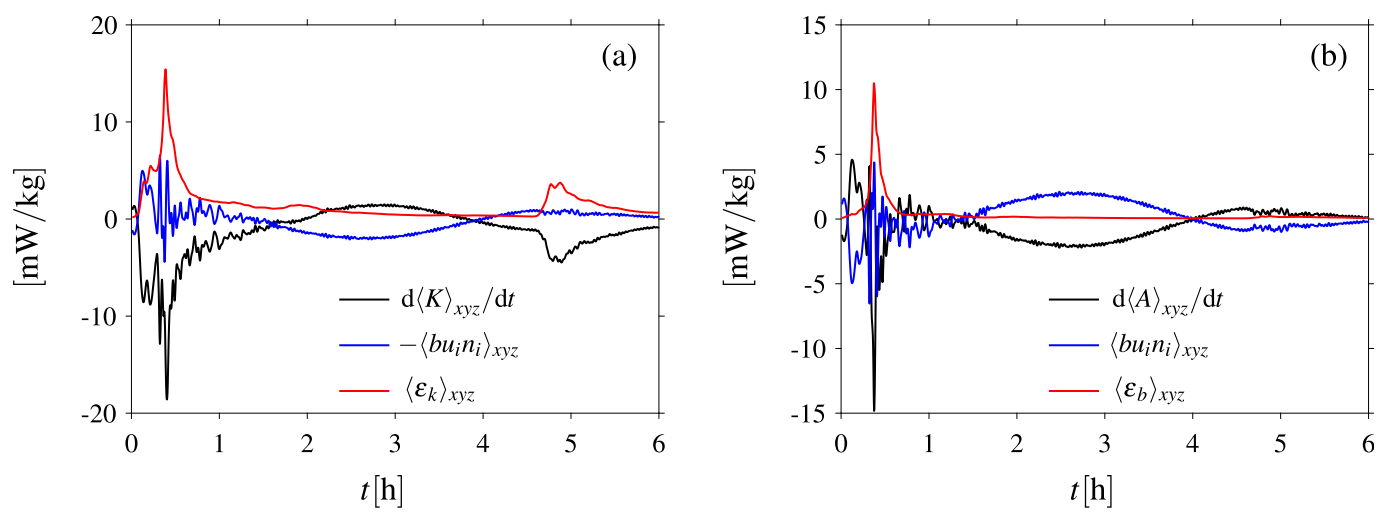

FIG. 11. Box-averaged (a) kinetic and (b) potential energy budget as defined in Eqs. (14) and (15).

that these power-laws should remain for even higher Reynolds number.

\section{e. Kinetic and potential energy}

Here, we leave the Reynolds number comparison aside and focus on the temporal evolution of the boxaveraged kinetic energy and available potential energy, as well as on the evolution of their individual components. We only show results for the high-Reynoldsnumber case IGW72, since the qualitative behavior for case IGW81 is essentially the same.

The box-averaged kinetic and potential energy evolve in time according to

$$
\frac{d\langle K\rangle_{x y z}}{d t}-\left\langle b u_{i} n_{i}\right\rangle_{x y z}+\left\langle\varepsilon_{k}\right\rangle_{x y z}=0
$$

and

$$
\frac{d\langle A\rangle_{x y z}}{d t}+\left\langle b u_{i} n_{i}\right\rangle_{x y z}+\left\langle\varepsilon_{b}\right\rangle_{x y z}=0
$$

Figure 11 shows each term of the equations above as a function of time. Not surprisingly, $d\langle K\rangle_{x y z} / d t$ and $d\langle A\rangle_{x y z} / d t$ are mostly negative, as it is expected for a decaying wave. This observation is in agreement with the previous flow-field visualizations, from which we have already seen that flow structures fade away and are less likely to be found toward the end of the simulation. Nevertheless, Fig. 11 shows that for certain time windows there is a surplus of energy that causes positive variations in either the kinetic or the potential energy. From Eqs. (14) and (15) it is clear that this can only occur when the transfer term $\left\langle b u_{i} n_{i}\right\rangle_{x y z}$ becomes larger than the energy dissipation rate. For the kinetic energy, Fig. 11a shows that this is the case for $2<t<4 \mathrm{~h}$ (approximately), whereas for the potential energy, Fig. $11 \mathrm{~b}$ shows positive variations of $d\langle A\rangle_{x y z} / d t$ within the interval $4<t<6 \mathrm{~h}$. Further, we also notice that during the breaking events, i.e., when dissipation is highest, potential energy is converted into kinetic energy, whereas the opposite prevails during calmer periods; to see that, contrast for example in Fig. 11 the behavior of $\left\langle b u_{i} n_{i}\right\rangle_{x y z}$ at $t \approx 0.5 \mathrm{~h}$ and $t \approx 5 \mathrm{~h}$ with its behavior at $t \approx 3 \mathrm{~h}$.

Now, we analyze the individual contributions of the three Cartesian velocity components to the kinetic energy by following in time $\langle u u\rangle_{x y z},\langle v v\rangle_{x y z}$, and $\langle w w\rangle_{x y z}$, and the buoyancy variance $\langle b b\rangle_{x y z} / N^{2}=2\langle A\rangle_{x y z}$. Results in Fig. 12 show that energy is mostly contained in the $\langle u u\rangle_{x y z}$ and $\langle v v\rangle_{x y z}$ components. In fact, both of them account for $99 \%$ of the total kinetic energy and approximately $69 \%$ of the total energy; the potential energy stores the remaining $31 \%$ of the total energy. If we focus on the first hour of the simulation, i.e., $t<$ $0.5 \mathrm{~h}$ (first breaking event), we observe that $\langle u u\rangle_{x y z}$ and $\langle v v\rangle_{x y z}$ decrease in magnitude, whereas $\langle w w\rangle_{x y z}$ and $\langle b b\rangle_{x y z} / N^{2}$ increase. This suggests that during the breaking events energy is transferred to $\langle w w\rangle_{x y z}$ and also stored as potential energy. Indeed, at the last breaking event, i.e., $t=4.88 \mathrm{~h},\langle b b\rangle_{x y z} / N^{2}$ rises once again, although less remarkable than during the first breaking event at $t=0.38 \mathrm{~h}$.

Regarding the cross correlations, Fig. 12 shows that $\langle u v\rangle_{x y z}$ is mostly positive, but undergoes a sign change during the third breaking event, $\langle u w\rangle_{x y z}$ is slightly negative throughout the whole simulation; $\langle v w\rangle_{x y z}$ on the other hand, fluctuates weakly around null (Fig. 12b).

\section{f. The energy transfer mechanism}

A more detailed notion of the energy transfer mechanism can be obtained through an energy budget analysis. To this end, we write the (componentwise) evolution equations for the kinetic and potential energy, which are obtained from Eqs. (1)-(3) upon multiplication with the corresponding velocity and buoyancy fields followed by a box average: 

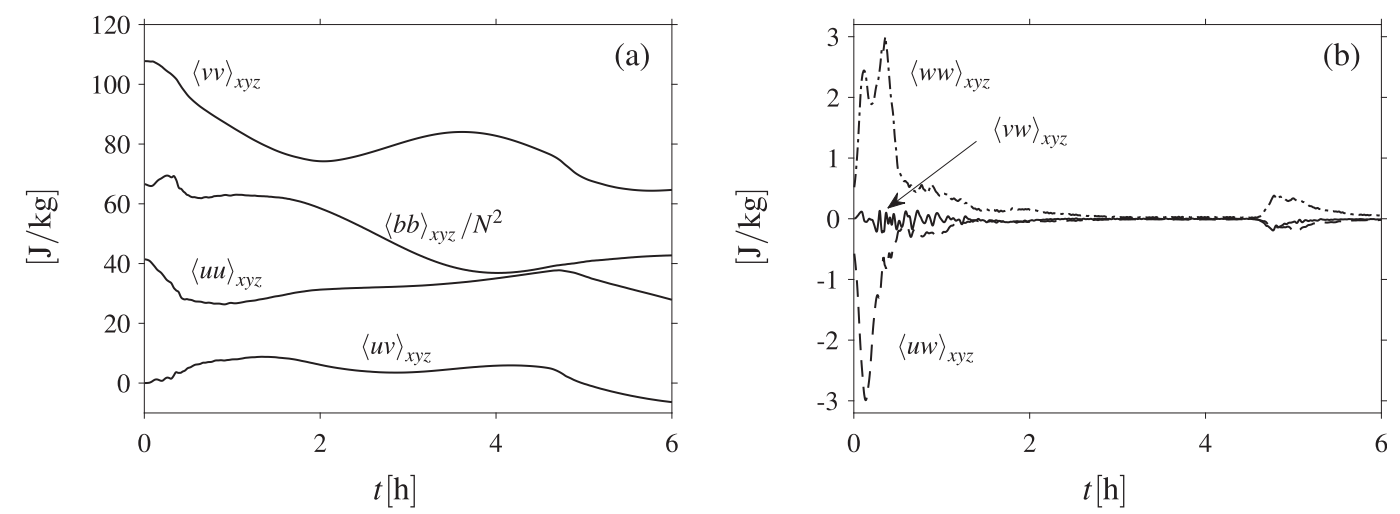

FIG. 12. Time evolution of the individual components of the kinetic and potential energy and the cross correlations for case IGW72. The data are split in (a) and (b) for better visibility.

$$
\begin{aligned}
\frac{\partial\left\langle u_{i} u_{i}\right\rangle_{x y z}}{\partial t}= & \left\langle T_{\text {pres,ii }}\right\rangle_{x y z}-\left\langle T_{\text {cor, }, \mathrm{i}}^{*}\right\rangle_{x y z} \\
& +\left\langle T_{\text {buoy,ii }}^{*}\right\rangle_{x y z}-\left\langle\varepsilon_{k, \mathrm{ii}}\right\rangle_{x y z}
\end{aligned}
$$

and

$$
\begin{aligned}
\frac{1}{N^{2}} \frac{\partial\langle b b\rangle_{x y z}}{\partial t}= & -\left\langle T_{\text {buoy, }, 11}\right\rangle_{x y z}-\left\langle T_{\text {buoy }, 22}\right\rangle_{x y z} \\
& -\left\langle T_{\text {buoy }, 33}\right\rangle_{x y z}-2\left\langle\varepsilon_{b}\right\rangle_{x y z}
\end{aligned}
$$

Equations (16) and (17) refer to the components of twice the kinetic and potential energy, respectively. In Eq. (16), no implicit summation over repeated indices is considered, and the subscript $i$ merely indicates the Cartesian direction, i.e., $i=\{1,2,3\}$ implies $\{x, y, z\}$. The terms on the right-hand side of Eqs. (16) and (17) represent the contributions due to the pressure field $\left(T_{\text {pres }}\right)$, the Coriolis force $\left(T_{\text {cor }}\right)$, the buoyancy force $\left(T_{\text {buoy }}\right)$ and the energy dissipation rate. Also, note that a superscript asterisk (*) appears in Eq. (16) to denote the terms that contain contributions from the pressure field, as explained next.

We isolate the influence due to the Coriolis and the buoyant forces in Eq. (16) by eliminating their contribution from the pressure field and adding it to their respective transfer terms. That is, first we split the pressure field $p$, which satisfies the Poisson equation

$$
\nabla^{2} p=-\nabla \cdot(\mathbf{u} \cdot \nabla \mathbf{u})-\nabla \cdot\left(f_{c} \mathbf{n} \times \mathbf{u}\right)+\nabla \cdot(b \mathbf{n}),
$$

such that $p=p_{\text {adv }}+p_{c}+p_{b}$, where $p_{\text {adv }}, p_{c}$, and $p_{b}$ are the solutions of Eq. (18) considering either the first, second, and third term on the right-hand side, respectively. Then, we include the effects of $p_{c}$ and $p_{b}$ to $T_{\mathrm{cor}, i}$ and $T_{\text {buoy }, i i}$, to form $\left\langle T_{\text {cor }, i i}^{*}\right\rangle_{x y z}$ and $\left\langle T_{\text {buoy }, i i}^{*}\right\rangle_{x y z}$. Hence, $\left\langle T_{\text {press }, i i}\right\rangle_{x y z}$ in Eq. (16) contains only the usual contribution due to $p_{\text {adv }}$. The individual pressure contributions in $\left\langle T_{\text {press }, i i}\right\rangle_{x y z},\left\langle T_{\text {cor }, i i}^{*}\right\rangle_{x y z}$, and $\left\langle T_{\text {buoy }, i i}^{*}\right\rangle_{x y z}$ do neither produce nor destroy kinetic energy, but rather redistribute energy between the three velocity components in such a way that the fluid volume is conserved (incompressible flow). The buoyancy term appears in Eq. (17) without an asterisk, since the pressure field does not play a role in this equation.

The evolution of each term is presented in Fig. 13. Figures 13a-c show the term-by-term energy budget for $i=\{1,2,3\}$ and Eq. (16), whereas Fig. 13d presents the results from Eq. (17). By individually analyzing the temporal evolution of each term, we find that $T_{\text {press }}$ is the main supplier of energy to $\langle w w\rangle_{x y z}$; this energy is transferred away from $\langle u u\rangle_{x y z}$, as clearly visible during the first breaking event, for which it is evident that $\left\langle T_{\text {press,11 }}\right\rangle$ is negative (Fig. 13a) and $\left\langle T_{\text {press,33}}\right\rangle$ is positive with roughly the same shape (Fig. 13c). We attribute this fact to the primary instability, which is predominantly aligned with the $x$ and $y$ directions and the main reason for the roll-up of eddies observed prior to the first breaking event.

Regarding the conversion from kinetic to potential energy, we see from Fig. 13d that $\left\langle T_{\text {buoy,33 }}\right\rangle_{x y z}$ is positive when the wave breaks, and the corresponding $\left\langle T_{\text {buoy,33 }}^{*}\right\rangle_{x y z}$ is essentially negative in Fig. 13c. Therefore, we conclude that most of the energy gained by $\langle w w\rangle_{x y z}$ through $\left\langle T_{\text {press, } 33}\right\rangle_{x y z}$ is converted into potential energy by $\left\langle T_{\text {buoy,33 }}\right\rangle_{x y z}$. The difference between the sink $\left\langle T_{\text {buoy, } 33}^{*}\right\rangle_{x y z}$ in the $\langle w w\rangle_{x y z}$ balance and $\left\langle T_{\text {buoy, } 33}\right\rangle_{x y z}$, which is a source for $\langle b b\rangle_{x y z} / N^{2}$, is due to the pressure contribution which transfers energy to the other components of the kinetic energy, in particular to $\langle u u\rangle_{x y z}$. In Fig. 14, we include a diagram that summarizes the transfer mechanism and the function/relevance of each term in Eqs. (16) and (17).

\section{g. Turbulent kinetic and potential energy}

So far, we have looked at the wave breakdown from a global sense, i.e., the velocity and buoyancy fields 

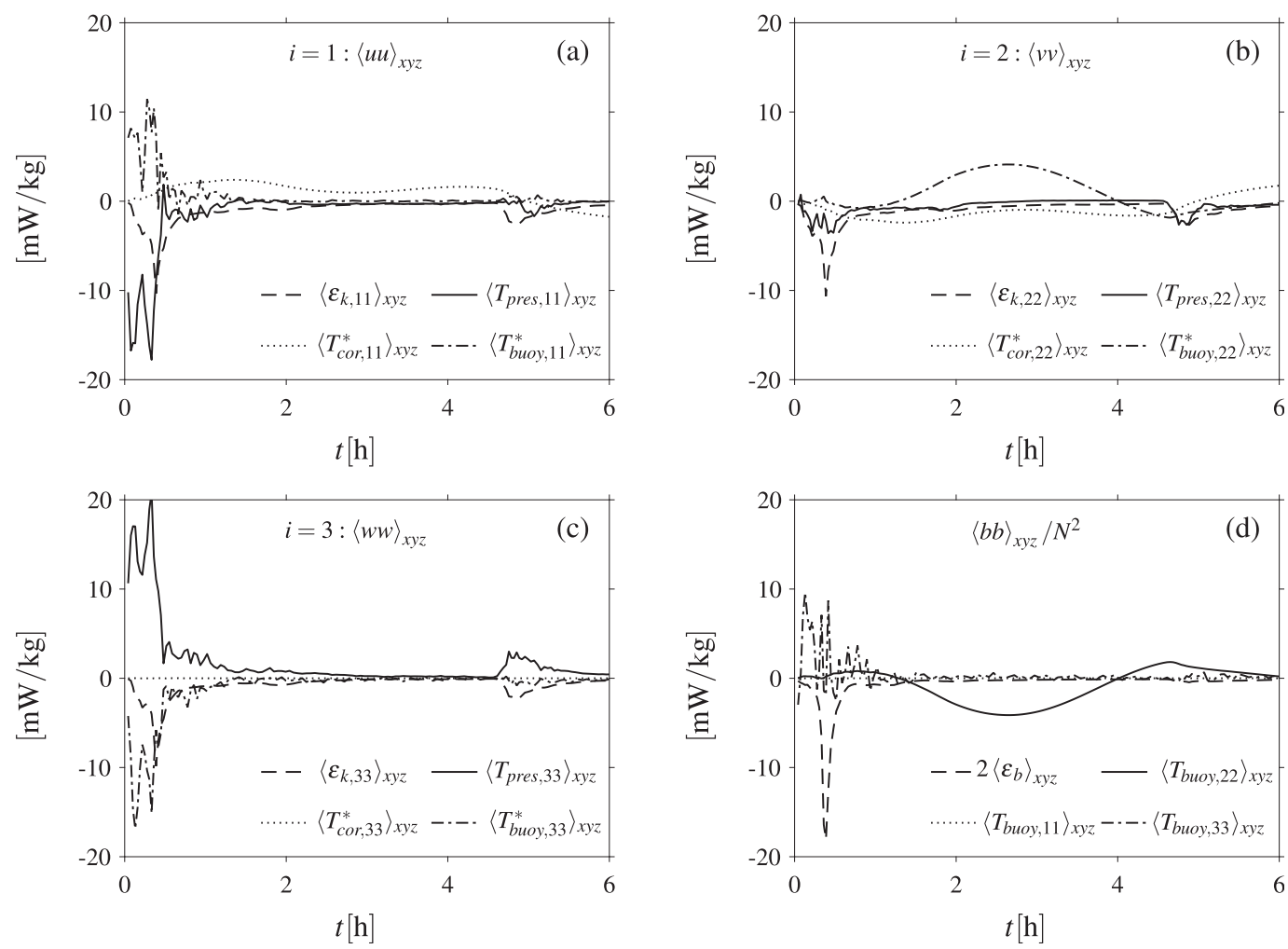

FIG. 13. Energy budget showing the time evolution of the contribution due to the Coriolis force $\left(T_{\text {cor }}\right)$, pressure ( $\left.T_{\text {pres }}\right)$, buoyancy $\left(T_{\text {buoy }}\right)$, and dissipation $\left(\left\langle\varepsilon_{k, \mathrm{ii}}\right\rangle_{x y z}\right)$ for case IGW72 with (a)-(c) $i=\{1,2,3\}$ in Eq. (16) and (d) the terms of Eq. (17).

represented both the decaying base wave and the turbulent structures. Although information about the global quantities are valuable for modeling approaches which do not resolve any scale of the wave, more refined models would probably aim at resolving at least the base wave and parameterizing only small-scale turbulent fluctuations. To this end, we decompose the velocity and the buoyancy field in two contributions: one related to the base wave and a second related to small-scale fluctuations.

To split the fields we first average them over $x-y$ planes and then compute the difference between the average and the local solution, e.g., the $x$ component of the velocity field is $u(x, y, z)=\langle u\rangle_{x y}(z)+u^{\prime}(x, y, z)$. Throughout time, the vertical profiles of the mean kinetic and mean potential energy of the large scales, i.e., $\left\langle u_{i}\right\rangle_{x y}\left\langle u_{i}\right\rangle_{x y} / 2$ and $\langle b\rangle_{x y}\langle b\rangle_{x y} /\left(2 N^{2}\right)$, remain similar to the profiles obtained with the full field (not shown). From the terms that constitute the mean kinetic energy, $\langle u\rangle_{x y}\langle u\rangle_{x y}$ is the one that changes the most in time (although modestly). In fact, we already saw in the previous section that this term is the one responsible for supplying energy to the third velocity component.

The temporal evolution of the fluctuation covariances, i.e., $\left\langle u^{\prime} u^{\prime}\right\rangle_{x y z},\left\langle v^{\prime} v^{\prime}\right\rangle_{x y z},\left\langle w^{\prime} w^{\prime}\right\rangle_{x y z}$, and $\left\langle b^{\prime} b^{\prime}\right\rangle_{x y z} / N^{2}$, shows that during the breaking events there is a significant increase of energy in the small-scale fluctuations, thus consistent with the idea that when the wave breaks, energy is extracted from the base wave (mean flow). This is clear from Fig. 15, which also shows that the energy gain by the fluctuations is more pronounced for the first breaking event, reinforcing that this event is indeed stronger than the others.

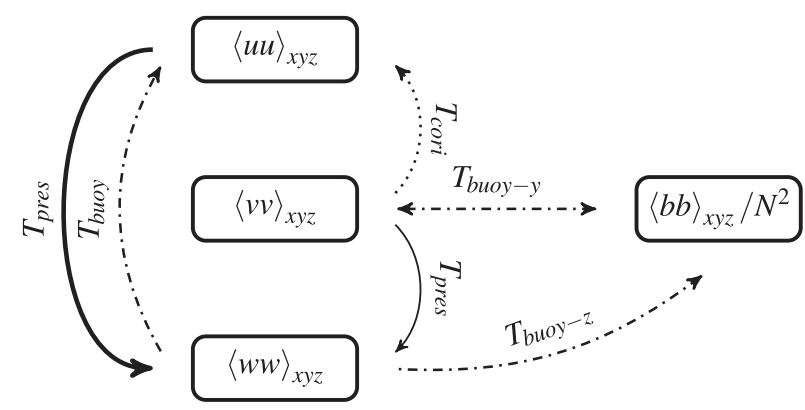

FIG. 14. Schematic of the energy transfer mechanism and the role of each term during the wave breaking event. Line styles are as in Fig. 13. The pressure term $\left(T_{\text {pres }}\right)$ appears as a thicker line to highlight its dominance over the other terms. Note that we have omitted the dissipation in the diagram, since its role is evident. 

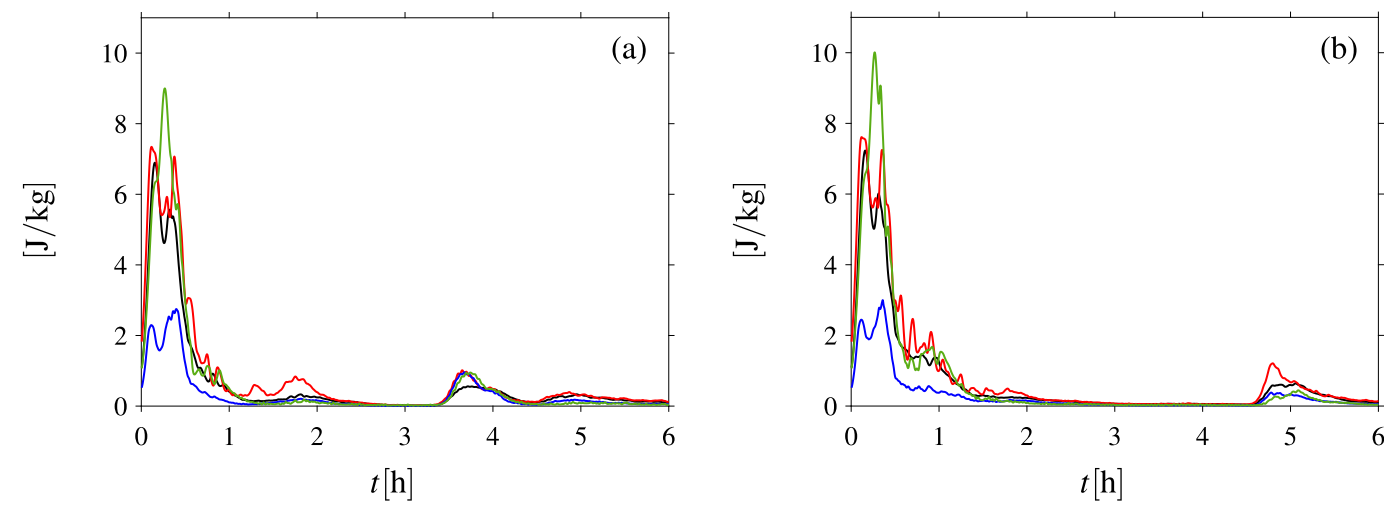

FIG. 15. Time evolution of the small-scale fluctuation covariances for (a) case IGW81 and (b) case IGW72. Different line colors denote the different terms: $\left\langle u^{\prime} u^{\prime}\right\rangle_{x y z}$ (black), $\left\langle v^{\prime} v^{\prime}\right\rangle_{x y z}$ (red), $\left\langle w^{\prime} w^{\prime}\right\rangle_{x y z}$ (blue), and $\left\langle b^{\prime} b^{\prime}\right\rangle_{x y z} / N^{2}$ (green).

The split of the velocity field allows us also to explore the energy transfer between the mean and the fluctuating field. Writing the governing equations for the energy of the mean and the fluctuating fields, one sees that they include coupling terms, namely, the production of kinetic and potential energy, which are sink terms for the mean kinetic and potential energy equation, and source terms in the corresponding equation for the energy of the fluctuations. The production of kinetic and potential energy are defined as

$$
\left\langle\mathscr{P}_{k}\right\rangle_{x y}=-\left\langle u_{i}^{\prime} u_{j}^{\prime}\right\rangle_{x y} \frac{\partial\left\langle u_{i}\right\rangle_{x y}}{\partial x_{j}}
$$

and

$$
\left\langle\mathscr{P}_{b}\right\rangle_{x y}=-\frac{1}{N^{2}}\left\langle b^{\prime} u_{j}^{\prime}\right\rangle_{x y} \frac{\partial\langle b\rangle_{x y}}{\partial x_{j}},
$$

respectively. The decomposition also yields the pseudo kinetic and potential energy dissipation rate, which are based on the fluctuations, and are given as

$$
\left\langle\varepsilon_{k}^{\prime}\right\rangle_{x y}=\nu\left\langle\frac{\partial u_{i}^{\prime}}{\partial x_{j}} \frac{\partial u_{i}^{\prime}}{\partial x_{j}}\right\rangle_{x y}
$$

and

$$
\left\langle\varepsilon_{b}^{\prime}\right\rangle_{x y}=\frac{\alpha}{N^{2}}\left\langle\frac{\partial b^{\prime}}{\partial x_{j}} \frac{\partial b^{\prime}}{\partial x_{j}}\right\rangle_{x y},
$$

respectively. Notice that the above quantities are all functions of the vertical coordinate $z$ and that $\left\langle\varepsilon_{k}^{\prime}\right\rangle_{x y}$ and $\left\langle\varepsilon_{b}^{\prime}\right\rangle_{x y}$ are positive quantities, whereas $\left\langle\mathscr{P}_{k}\right\rangle_{x y}$ and $\left\langle\mathscr{P}_{b}\right\rangle_{x y}$ can assume both positive and negative values. The complete set of equations including all terms that contribute to the evolution of the mean and the fluctuating part can be found in Achatz (2007b). We do not include them here, since the other terms do not contribute to the energy exchange between the horizontal mean and the horizontally dependent deviations.

Figures 16 and 17 show the production of kinetic and potential energy for cases IGW81 and IGW72 normalized by the corresponding pseudo energy dissipation rate. From Fig. 16, we see that the in upper half of the domain, the ratio $\left\langle\mathscr{P}_{k}\right\rangle_{x y} /\left\langle\varepsilon_{k}^{\prime}\right\rangle_{x y}$ is approximately unity (cf. the zoomed panels). In fact, for the first breaking event of case IGW81 (Fig. 16a) the ratio is 1.12 when averaged in the range $1500<z<3000 \mathrm{~m}$, and for the last breaking event of case IGW72 (Fig. 16d) the averaged ratio in the interval $1500<z<2500 \mathrm{~m}$ is 0.97 . Conversely, we do not identify any sign of a local balance between the production and the dissipation of potential energy (Fig. 17); although we only show the results at the first breakdown, similar results are obtained for the other breaking events.

These figures also reveal that the vertical profiles of $\left\langle\mathscr{P}_{k}\right\rangle_{x y}$ and $\left\langle\mathscr{P}_{b}\right\rangle_{x y}$ at the wave breakdown are inhomogeneous distributed in space. The fact that we find both positive as well as negative values of $\left\langle\mathscr{P}_{k}\right\rangle_{x y}$ and $\left\langle\mathscr{P}_{b}\right\rangle_{x y}$ indicates that energy transfers occur from the mean field to the fluctuations, and vice versa. Specifically, for the kinetic energy, we find that $\left\langle\mathscr{P}_{k}\right\rangle_{x y}$ is predominantly positive in the upper half of the numerical domain $(z>$ $1500 \mathrm{~m})$. The large ratios of $\left\langle\mathscr{P}_{k}\right\rangle_{x y} /\left\langle\varepsilon_{k}^{\prime}\right\rangle_{x y}$ observed in Figs. $16 \mathrm{~b}$ and $16 \mathrm{~d}$ are a consequence of the low energy dissipation rate, as already observed in the Hovmöller diagrams in section $3 \mathrm{c}$.

\section{Conclusions}

We have presented results from fully resolved threedimensional simulations of a inertia-gravity wave breaking in the middle atmosphere. Two cases were considered, 
(a)

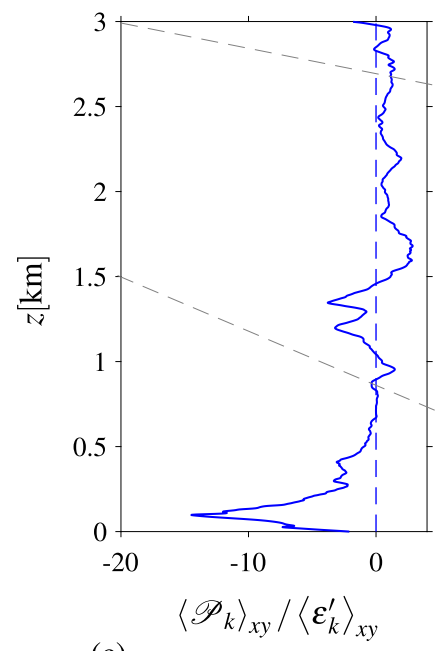

(c)

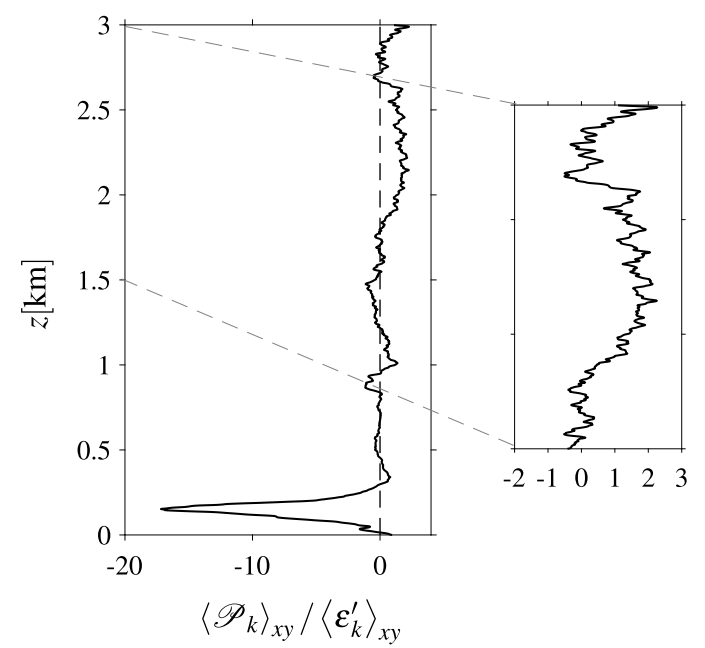

(b)

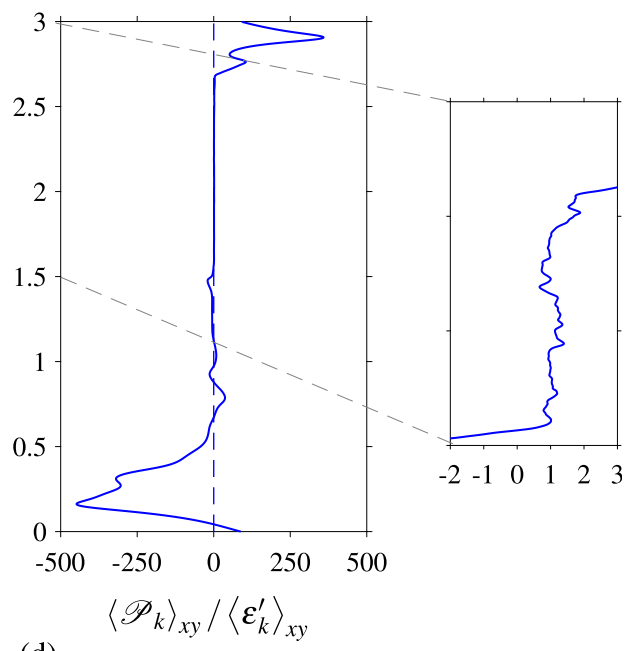

(d)

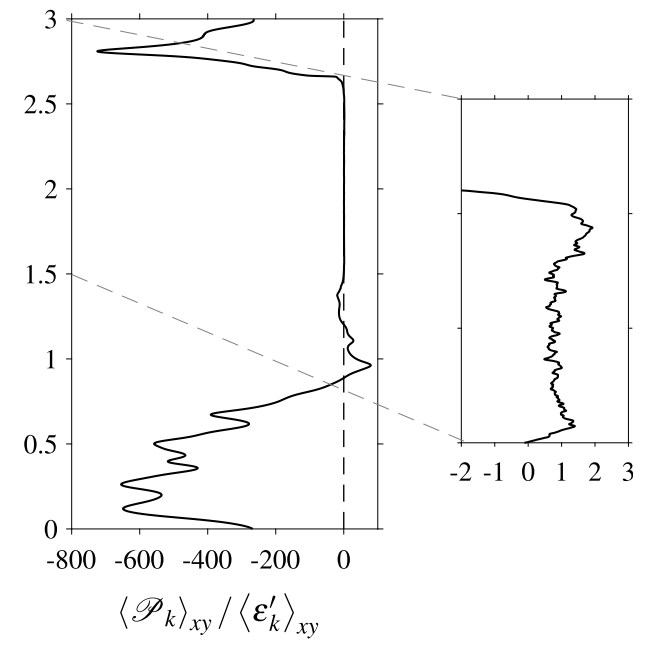

FIG. 16. Vertical profiles of the ratio between the kinetic energy production and the (pseudo) kinetic energy dissipation rate for (a),(b) case IGW81 and (c),(d) case IGW72, corresponding to the (a),(c) first and (b),(d) last breaking events.

which correspond to two different Reynolds numbers and, accordingly, two different geopotential altitudes.

Both cases IGW81 and IGW72 undergo a sequence of breaking events, which are marked by a rise in the kinetic energy dissipation rate. The general picture of the wave breakdown is somewhat similar for both Reynolds numbers. Flow-field visualization illustrates the outbreak of fine scales of motion in the vertical upper half of the numerical domain, which are finer in case IGW72 (higher Reynolds number). Along the same line, Hovmöller diagrams show that the kinetic energy dissipation rate is more intense in the upper half of the domain, signalizing the dominance of large spatial velocity gradients where the base wave is least stable. The Richardson number can be used as a diagnostic tool to detect these regions.
At the time of the wave breakdown, the kinetic energy dissipation rate tensor exhibits an equipartition of dissipation among its individual components, and the vertical kinetic energy spectrum displays a $5 / 3$ scaling for a broad range of wavelengths. At periods in-between the breaking events, on the other hand, the kinetic energy dissipation rate tensor is strongly anisotropic and the vertical kinetic energy spectrum scales with a cubic power law, indicating the relevance of buoyancy effects at these times.

Through an energy budget analysis we saw that the energy transfer mechanism during the wave breakdown is dominated by the energy transfer from $\langle u u\rangle_{x y z}$ to $\langle w w\rangle_{x y z}$. This was shown to be due to the pressure term, which drives the perturbation field toward an isotropic 

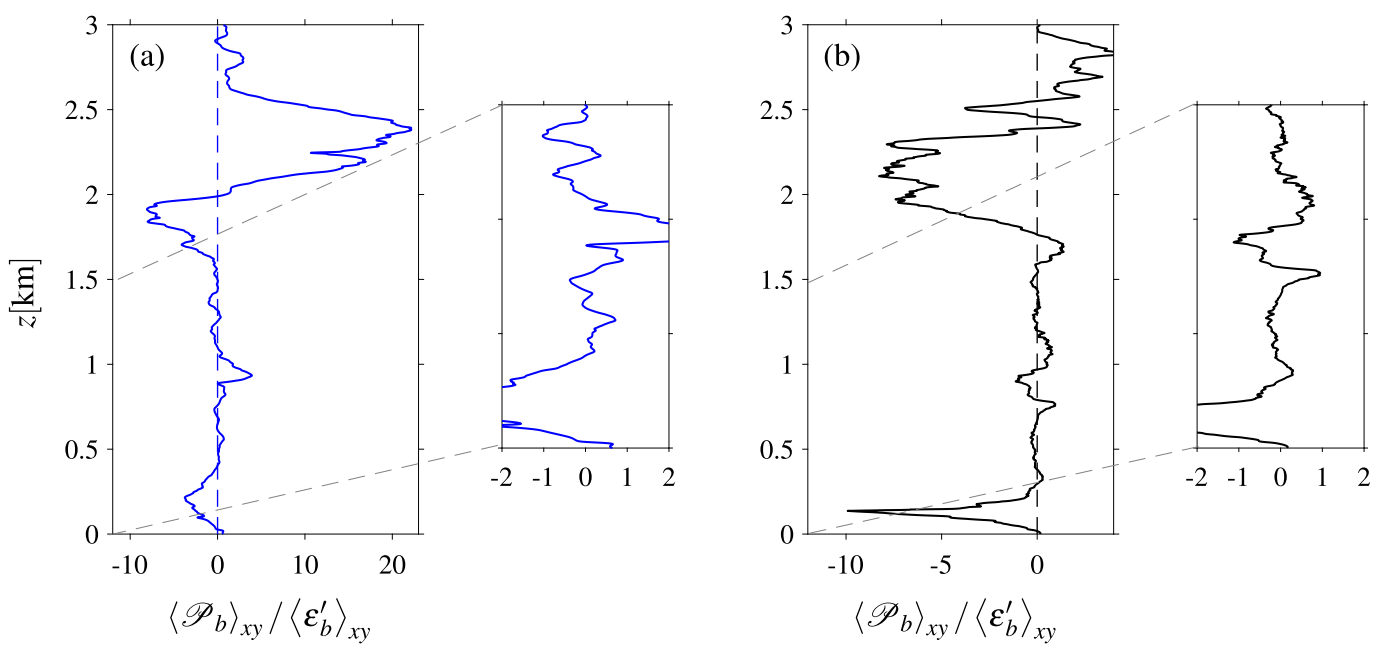

FIG. 17. Vertical profiles of the ratio between the potential energy production and the (pseudo) potential energy dissipation rate for (a) case IGW81 and for (b) case IGW72. The panels correspond to the first breaking event in both cases.

state while ensuring volume conservation, and due to the subsequent conversion into potential energy. The role of the Coriolis force, although less relevant, was also present in shifting energy from $\langle v v\rangle_{x y z}$ to $\langle u u\rangle_{x y z}$.

Complementing these observations, we also decomposed the velocity and the buoyancy fields into a mean and a fluctuating part. These results show that the local transfer of kinetic energy between the mean and the fluctuating parts is mainly forward, i.e., from the mean to the fluctuations. In the upper half of the numerical domain, where finer scales of motion were identified, we found that the pseudo kinetic energy dissipation rate and the kinetic energy production are roughly in balance during the breaking events.

Acknowledgments. The authors are grateful to three anonymous referees whose suggestions have improved this manuscript. The simulations have been performed at the Dutch National Supercomputer Cartesius with the support of the SURF cooperative and the Dutch Research Council (NWO) Project 16108. The authors thank the technical expertise and the assistance provided by SURFsara. The authors report no conflict of interest.

\section{REFERENCES}

Achatz, U., 2005: On the role of optimal perturbations in the instability of monochromatic gravity waves. Phys. Fluids, 17, 094107, https://doi.org/10.1063/1.2046709.

_ 2007a: Modal and nonmodal perturbations of monochromatic high-frequency gravity waves: Primary nonlinear dynamics. J. Atmos. Sci., 64, 1977-1994, https://doi.org/10.1175/JAS3940.1.

- 2007b: The primary nonlinear dynamics of modal and nonmodal perturbations of monochromatic inertia-gravity waves. J. Atmos. Sci., 64, 74-95, https://doi.org/10.1175/JAS3827.1.
_- , and G. Schmitz, 2006: Shear and static instability of inertia-gravity wave packets: Short-term modal and nonmodal growth. J. Atmos. Sci., 63, 397-413, https://doi.org/ 10.1175/JAS3636.1.

Andreassen, Ø., C. E. Wasberg, D. C. Fritts, and J. R. Isler, 1994: Gravity wave breaking in two and three dimensions: 1 . Model description and comparison of two-dimensional evolutions. J. Geophys. Res., 99, 8095, https://doi.org/10.1029/93JD03435.

Bauer, P., A. Thorpe, and G. Brunet, 2015: The quiet revolution of numerical weather prediction. Nature, 525, 47-55, https:// doi.org/10.1038/nature14956.

Berner, J., and Coauthors, 2017: Stochastic parameterization: Toward a new view of weather and climate models. Bull. Amer. Meteor. Soc., 98, 565-588, https://doi.org/10.1175/ BAMS-D-15-00268.1.

Brethouwer, G., P. Billant, E. Lindborg, and J.-M. Chomaz, 2007: Scaling analysis and simulation of strongly stratified turbulent flows. J. Fluid Mech., 585, 343-368, https://doi.org/10.1017/ S0022112007006854.

Fritts, D. C., and L. Wang, 2013: Gravity wave-fine structure interactions. Part II: Energy dissipation evolutions, statistics, and implications. J. Atmos. Sci., 70, 3735-3755, https://doi.org/ 10.1175/JAS-D-13-059.1.

_, J. R. Isler, and Ø. Andreassen, 1994: Gravity wave breaking in two and three dimensions: 2 . Three-dimensional evolution and instability structure. J. Geophys. Res., 99, 8109, https:// doi.org/10.1029/93JD03436.

—_ L. Wang, J. Werne, T. Lund, and K. Wan, 2009a: Gravity wave instability dynamics at high Reynolds numbers. Part I: Wave field evolution at large amplitudes and high frequencies. J. Atmos. Sci., 66, 1126-1148, https://doi.org/ 10.1175/2008JAS2726.1.

$-, \ldots,-, \ldots$, and $-2009 \mathrm{~b}$ : Gravity wave instability dynamics at high Reynolds numbers. Part II: Turbulence evolution, structure, and anisotropy. J. Atmos. Sci., 66, 11491171, https://doi.org/10.1175/2008JAS2727.1.

,-- , and - , 2013: Gravity wave-fine structure interactions. Part I: Influences of fine structure form and orientation on flow evolution and instability. J. Atmos. Sci., 70, 3710-3734, https://doi.org/10.1175/JAS-D-13-055.1. 
Fruman, M. D., and U. Achatz, 2012: Secondary instabilities in breaking inertia-gravity waves. J. Atmos. Sci., 69, 303-322, https://doi.org/10.1175/JAS-D-10-05027.1.

- - S. Remmler, U. Achatz, and S. Hickel, 2014: On the construction of a direct numerical simulation of a breaking inertia-gravity wave in the upper mesosphere. J. Geophys. Res. Atmos., 119, $11613-11640$, https://doi.org/10.1002/ $2014 \mathrm{jd} 022046$.

Hines, C. O., 1997: Doppler-spread parameterization of gravitywave momentum deposition in the middle atmosphere. Part 1: Basic formulation. J. Atmos. Sol.-Terr. Phys., 59, 371-386, https://doi.org/10.1016/S1364-6826(96)00079-X.

Isler, J. R., D. C. Fritts, Ø. Andreassen, and C. E. Wasberg, 1994: Gravity wave breaking in two and three dimensions: 3. Vortex breakdown and transition to isotropy. J. Geophys. Res., 99, 8125, https://doi.org/10.1029/93JD03437.

Kaneda, Y., T. Ishihara, M. Yokokawa, K. Itakura, and A. Uno, 2003: Energy dissipation rate and energy spectrum in high resolution direct numerical simulations of turbulence in a periodic box. Phys. Fluids, 15, L21-L24, https://doi.org/ 10.1063/1.1539855.

Lindzen, R. S., 1981: Turbulence and stress owing to gravity wave and tidal breakdown. J. Geophys. Res., 86, 9707, https:// doi.org/10.1029/JC086iC10p09707.
Pekurovsky, D., 2012: P3DFFT: A framework for parallel computations of Fourier transforms in three dimensions. SIAM J. Sci. Comput., 34, C192-C209, https://doi.org/10.1137/11082748X.

Pestana, T., and S. Hickel, 2019: Regime transition in the energy cascade of rotating turbulence. Phys. Rev., 99E, 053103, https://doi.org/10.1103/physreve.99.053103.

Remmler, S., and S. Hickel, 2013: Spectral structure of stratified turbulence: Direct numerical simulations and predictions by large eddy simulation. Theor. Comput. Fluid Dyn., 27, 319336, https://doi.org/10.1007/s00162-012-0259-9.

—, M. D. Fruman, and S. Hickel, 2013: Direct numerical simulation of a breaking inertia-gravity wave. J. Fluid Mech., 722, 424-436, https://doi.org/10.1017/jfm.2013.108.

- S. Hickel, M. D. Fruman, and U. Achatz, 2015: Validation of large-eddy simulation methods for gravity wave breaking. J. Atmos. Sci., 72, 3537-3562, https://doi.org/10.1175/JAS-D14-0321.1.

Rogallo, R. S., 1977: An ILLIAC program for the numerical simulation of homogeneous incompressible turbulence. NASA Tech. Memo. 73, 203 pp., https://ntrs.nasa.gov/archive/nasa/ casi.ntrs.nasa.gov/19780005424.pdf.

Yau, K.-H., G. P. Klaassen, and L. J. Sonmor, 2004: Principal instabilities of large amplitude inertio-gravity waves. Phys. Fluids, 16, 936-951, https://doi.org/10.1063/1.1643916. 